\title{
Les productions à caractère symbolique du site moustérien de La Roche-Cotard à Langeais (Indre-et-Loire, France) dans leur contexte géologique
}

Symbolic character productions in the mousterian site of La Roche-Cotard in Langeais (Indre-et-Loire, France) and their geological context

Jean-Claude Marquet, Michel Lorblanchet, Yves Egels, José EsquerrePourtère et Maria-Sibylla Hesse

\section{OpenEdition Journals}

Édition électronique

URL : http://journals.openedition.org/paleo/2780

DOI : $10.4000 /$ paleo. 2780

ISSN : 2101-0420

Éditeur

SAMRA

Édition imprimée

Date de publication : 28 décembre 2014

Pagination : 169-194

ISSN : 1145-3370

Référence électronique

Jean-Claude Marquet, Michel Lorblanchet, Yves Egels, José Esquerre-Pourtère et Maria-Sibylla Hesse, " Les productions à caractère symbolique du site moustérien de La Roche-Cotard à Langeais (Indreet-Loire, France) dans leur contexte géologique », PALEO [En ligne], 25 | 2014, mis en ligne le 28 juillet 2015, consulté le 07 juillet 2020. URL : http://journals.openedition.org/paleo/2780 ; DOI : https:// doi.org/10.4000/paleo.2780

\section{(c)}

PALEO est mis à disposition selon les termes de la licence Creative Commons Attribution - Pas d'Utilisation Commerciale - Pas de Modification 4.0 International. 


\title{
Les productions à caractère symbolique du site moustérien de La Roche-Cotard à Langeais (Indre-et-Loire, France) dans leur contexte géologique
}

\author{
Jean-Claude MARQUET(1), Michel LORBLANCHET(2) \\ avec la collaboration de Yves EGELS ${ }^{(3)}$, José ESQUERRE-POURTÈRE(4), \\ Maria-Sibylla HESSE ${ }^{(5)}$
}

Résumé : Le site de La Roche-Cotard se trouve sur la rive droite de la vallée de la Loire à une vingtaine de kilomètres en aval de Tours. Le gisement, mis au jour en 1846, suite à l'extraction de matériaux, a été fouillé en 1912. Les recherches ont repris de 1975 à 1978, puis à partir de 2008.

Les premières recherches, en 1975, permettent la découverte, devant l'entrée de la grotte, d'un lambeau de plage de Loire sur lequel les hommes de Néandertal ont allumé un feu, abandonné des pièces d'industrie lithique et une protofigurine, appelée par M. Lorblanchet « masque de La Roche-Cotard » (Marquet, Lorblanchet 2000, 2003). Un abri très bas est également découvert ; il contient un peu d'industrie lithique et un aménagement de blocs au sol.

À partir de 2008, les nouvelles fouilles mettent au jour une petite grotte-abri comblée de sédiments, présentant également des indices d'occupation par l'homme de Néandertal. Elles poursuivent également le travail à l'intérieur de la cavité principale.

L'examen approfondi des parois, effectué par J.-C. Marquet avec les appuis de M. Lorblanchet, P. Paillet et E. ManEstier, permet de reconnaître quatre panneaux, trois de tracés digitaux et un de ponctuations. Ces traces sont visibles car il y a eu enlèvement de matière (au doigt ou à l'aide d'un instrument pouvant donner le même type de trace) sur la paroi de " craie tuffeau $"^{6}$ altérée, recouverte par un mince film de limon argileux. Sur l'un des panneaux, on observe une figuration circulaire, sur les deux autres des ensembles de tracés parallèles, organisés et rythmés. Quatre taches ocre rouge ont été repérées.

Les études géométrique, granulométrique et morphologique des dépôts, ainsi que les datations des os par le ${ }^{14} \mathrm{C}$ et des sédiments par la méthode OSL, montrent qu'après l'occupation par les hommes de Néandertal, l'entrée de la cavité a été occultée, pour n'être réouverte qu'en 1846 (voire seulement en 1912).

Aucune datation directe des tracés n'est possible. Cependant, leur altération locale, les datations des os et des sédiments situés juste devant les panneaux et naturellement celle de la fermeture de l'entrée de la grotte après l'occupation par Néandertal autorisent à exclure un âge récent.

Masque, tracés et traces de peinture dans une cavité inaccessible à Homo sapiens jusqu'en 1846, géométrie des dépôts, confèrent au site de La Roche-Cotard un très grand intérêt au moment où est sérieusement reconsidérée la question des compétences de l'homme de Néandertal.

(1) Chercheur associé, Laboratoire Archéologie et Territoires Tours, UMR 7324 CITERES, 16, Place Richemont, FR-37550 Saint-Avertin - jcmarquet@wanadoo.fr

(2) Directeur de recherches honoraire au CNRS - michel.lorblanchet@ wanadoo.fr

(3) École Nationale des Sciences Géographiques, Champs-sur-Marne - yves.egels@free.fr

(4) Professeur de S.V.T., FR-59239 Thumeries - jose.esquerre-pourtere@wanadoo.fr

(5) Professeure d'Histoire et de l'Histoire de l'art à l'École Waldorf de Potsdam (Allemagne) - sibylla.hesse@posteo.de

(6) Nom vernaculaire donné à cette roche. 
Mots-clés : tracés digitaux, art pariétal, ocre rouge, symbolique, Paléolithique moyen, Moustérien, Néandertal, Touraine.

\begin{abstract}
Symbolic character productions in the mousterian site of La Roche-Cotard in Langeais (Indre-et-Loire, France) and their geological context. La Roche-Cotard site is located on the right side of the Loire valley, about $20 \mathrm{~km}$ down-river from Tours. The site, uncovered in 1846, following the extraction of materials, was excavated in 1912. Research was taken up again from 1975 to 1978, then from 2008 onwards.

The first reprise of excavations in 1975 allowed for the discovery, at the entrance of a cave on a beach of the Loire on which Neanderthals have made fire, of stone tools and a proto-figurine that was named by M. Lorblanchet the "Mask of La Roche-Cotard" (Marquet \& Lorblanchet, 2000, 2003). A very low shelter was also discovered, which contains some stone tools and a layout of blocks on the ground.

The second reprise of excavations in 2008 allowed for the discovery of a small cave-shelter filled with sediments, presenting also indications of occupation by Neanderthals; but above all it enabled further work on the inside of the main cavity.

Deeper examination of the cave walls, carried out by J.-C. Marquet, with the support of M. Lorblanchet, P. Paillet and E. Man-Estier, allowed for the recognition of three panels of finger flutings and a panel of point-marks. These marks are visible due to the removal of matter (by hand or with the aid of an instrument giving the same type of marks) on the weathered «tuffeau chalk» wall, recovered by a thin film of silty clay. On one of the panels can be observed a circular representation, on the other two, sets of parallel markings, organized and rhythmic. Four red ochre stains were also discovered.

Geometric, granulometric and morphologic studies of the deposits, as well as ${ }^{14} \mathrm{C}$ datings of bones and datings of sediments through the OSL method, show that following the occupation by Neanderthals, the entrance to the cave was blotted out and was only reopened in 1846, or even in 1912.

No direct dating of the markings is possible. However, their localized weathering, datings of bones and sediments found just in front of the panels and naturally the closure of the cave's entrance after the occupation by Neanderthals allows us to rule out the possibility of a recent age.

Mask, markings and traces of painting in a cavity inaccessible to Homo sapiens until 1846, geometry of deposits, all add to the great interest in La Roche-Cotard site, at the very moment when we reconsider seriously the question of the competences of the Neanderthals.
\end{abstract}

Key-words: finger flutings, rock art, red ochre, symbol, Middle Palaeolithic, Neanderthal, Mousterian, Touraine.

Le site de La Roche-Cotard (LRC) se trouve sur la commune de Langeais en Indre-et-Loire. II se développe à mi-hauteur du versant en rive droite de la vallée de la Loire un peu en amont de la ville et à l'ouest de Cinq-Mars-laPile. Il est donc exposé au sud ; il se compose d'une grotte, d'une station en pied de falaise, d'un petit abri très bas et d'une grotte-abri de faibles dimensions. II n'est pas impossible que la connaissance que nous en avons aujourd'hui soit incomplète du fait de son contexte géologique et des circonstances très particulières qui ont présidé à sa découverte. Alors qu'une couche moustérienne a livré le masque de La Roche-Cotard, objet que beaucoup de spécialistes considèrent aujourd'hui comme une production à caractère symbolique de l'homme de Néandertal, et que diverses découvertes récentes enrichissent la question des compétences du prédécesseur de l'homme moderne en Europe occidentale, il convenait de porter à la connaissance de la communauté scientifique d'autres productions de ce site qui seront examinées dans cette note.

\section{1 - Historique de la découverte et des recherches sur le site}

Jusqu'au milieu du $\mathrm{XIX}^{\mathrm{e}}$ siècle et pendant un certain nombre de millénaires qui précèdent, le gisement est complètement masqué par des dépôts de versant dont on peut connaître la géométrie grâce aux profils naturels visibles à quelques centaines de mètres en aval et en amont du site.

En 1846, la mise en place de la voie ferrée de Tours à Angers demande des quantités colossales de matériaux que l'on va prendre au pied du versant de la vallée la plus proche de la portion à surélever. C'est ainsi que dans le parc actuel de la propriété de La Roche-Cotard (fig. 1), des masses importantes de sédiments ont été prélevées, en sapant le versant et s'avançant plus ou moins loin vers le rebord du plateau. L'entrée de la grotte est peut-être dégagée mais n'est pas alors vraiment découverte ou, si elle l'a été, aucun intérêt ne lui est apporté par les terrassiers qui chargeaient les tombereaux de terre.

C'est peut-être parce que la cavité se trouve à l'intérieur d'une propriété privée clôturée de murs que sa véritable découverte va tarder. En effet, François d'Achon, le propriétaire du lieu pénètre dans la grotte en janvier 1912 (d'Achon 1913). Avec ses ouvriers, il fouille l'essentiel du contenu en quelques semaines et trouve de nombreux ossements et des silex taillés (nous utiliserons le terme silex pour le matériau siliceux introduit sur le site et taillé), surtout devant l'entrée et dans la première partie de la cavité.

De nouvelles recherches sont entreprises dans les années 1970 , en trois campagnes, 1975, 1976 et 1977. Notre 


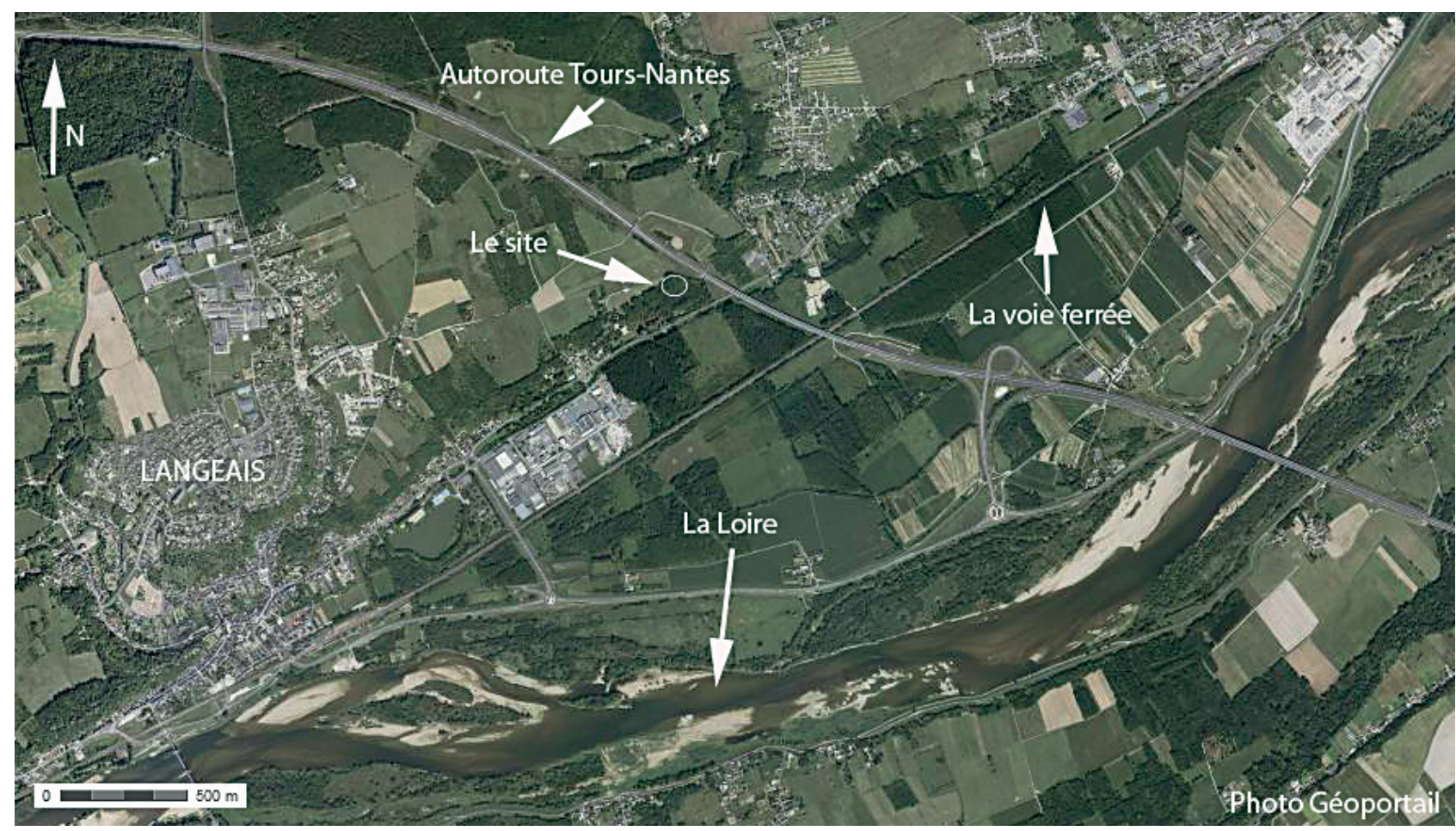

Figure 1 - La Roche-Cotard à Langeais en Indre-et-Loire. Situation géographique.

Figure 1 - La Roche-Cotard in Langeais, Indre-et-Loire. Geographical location.

objectif était alors double : identifier l'existence de lambeaux de couches à l'intérieur de la cavité et effectuer un sondage devant l'entrée de la grotte à la recherche d'éventuels niveaux d'occupation en place

Les trois campagnes de fouille donnent des résultats extrêmement intéressants (Marquet 1979, 1997) (fig. 2), qui se résument en :

- la découverte de lambeaux de couches, quelquefois assez conséquents, en place, dans la grotte F. d'Achon (LRC I) ;
- la découverte d'un niveau moustérien intact dans la fouille conduite en avant de l'entrée de la grotte (LRC II) ; c'est ce dernier qui a livré le masque de La Roche-Cotard ;

- celle, enfin, un peu plus à l'est, d'un abri très bas (LRC III) colmaté de sédiments (fig. 2).

Entre 1975 et 1977, le travail à l'intérieur de la cavité (LRC I) avait été peu poussé mais nous avions cependant examiné les parois. L'existence d'un film très mince de sédiment avait été observé ainsi que le très curieux «retrait» naturel de la paroi depuis sa base jusqu'à une certaine hauteur, toujours constante, dans toute la première moitié de la grotte. Enfin,

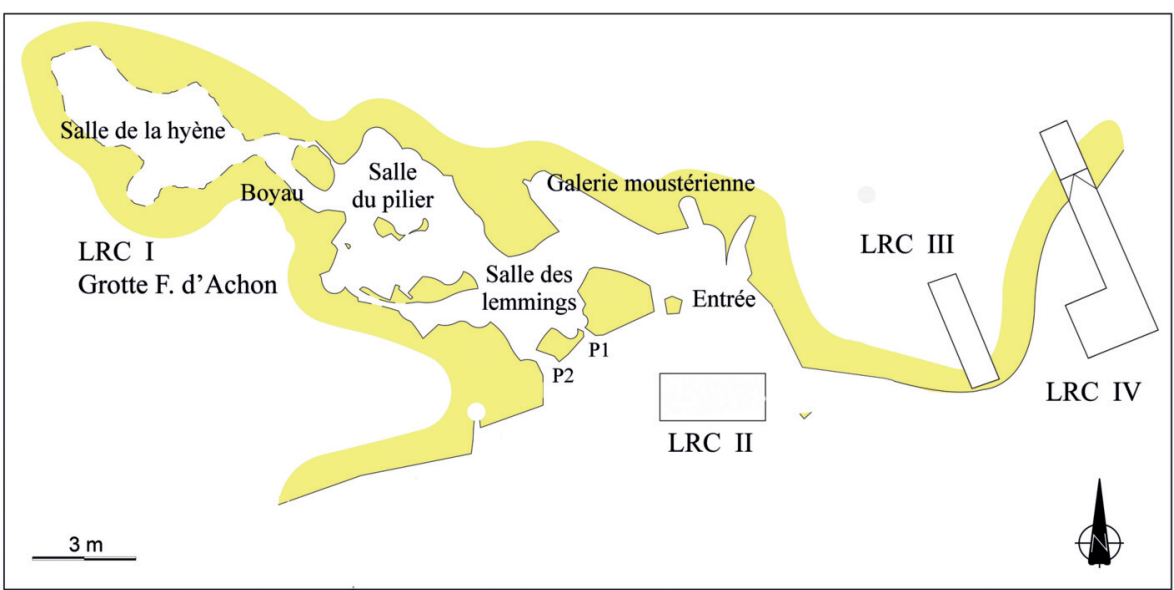

Figure 2 - La Roche-Cotard. Situation des quatre locus.

Figure 2 - La Roche-Cotard. Situation of the four locus of the site. 
avait été notée la présence de traces de toutes sortes sur les parois dont certaines semblaient avoir été faites au doigt.

À partir de 2008, de nouvelles recherches ont mis en évidence, plus à l'est, une petite grotte-abri masquée par une imposante succession de strates de 10 mètres de hauteur dans LRC IV (Ajas 2011). Elles ont surtout permis de reprendre l'étude des parois de la grotte d'Achon et de faire le relevé d'un certain nombre de traces, objets de cette publication. Deux séries de datations ont par ailleurs été réalisées, l'une par la méthode du ${ }^{14} \mathrm{C}$ sur des os, l'autre par la méthode OSL (Luminescence stimulée optiquement) sur des sédiments sableux.

\section{2 - Les différentes séquences stratigraphiques}

Pour les besoins de la démonstration que nous souhaitons faire ici, essentielle quant à la datation des tracés digitaux, il convient de présenter deux des différentes séquences stratigraphiques, celle de LRC I et celle de LRC IV.

\section{1 - La stratigraphie de La Roche-Cotard I}

En 1912, François d'Achon, avec l'aide de ses ouvriers, vide la grotte en quelques semaines pour y récupérer les silex taillés et les os des grands mammifères (d'Achon ibid). Dans sa publication, il donne un plan de la grotte avec, sommairement, l'emplacement des différentes trouvailles (fig. 3), des photos médiocres des os et des silex taillés exhumés mais malheureusement, pas de coupe stratigraphique du remplissage.

Lors de notre reprise des fouilles (1975-1977), un nouveau plan est levé, il sera repris plus tard par le service topographique du Syndicat des cavités 37 (fig. 2) au moment des travaux des fouilles de 2008. Nous fouillons alors en partie des lambeaux de couches résiduelles situés altitudinalement, les sédiments sont analysés.

On peut aujourd'hui considérer que dans la première partie de la cavité, la stratigraphie se décompose de la manière suivante (fig. 4):

- une couche inférieure, dont l'épaisseur est difficile à évaluer du fait de l'irrégularité du sol qui remonte très rapidement lorsque l'on s'éloigne de l'entrée. Elle est constituée d'un sédiment sableux très fin contenant quelques fragments osseux et pièces lithiques d'un silex de très belle qualité pour sa taillabilité et dans un excellent état de fraîcheur. Ce niveau a été daté par le ${ }^{14} \mathrm{C}$ dans la galerie moustérienne de 38060 ans \pm 940 (Lyon 7864) (tabl. 1) ; - une couche moyenne épaisse, composée d'un sédiment qui a les caractéristiques d'un limon de débordement de la Loire (Macaire in Marquet 1997). Elle contenait des restes de grands mammifères, des bovidés, des équidés, des cervidés, etc. (Byrne, Delpech, Prat in Marquet 1997) très fortement marqués par l'action des hyènes (os cassés et rongés), mais aucun d'industrie lithique. Elle a été datée par le ${ }^{14} \mathrm{C}$ d'un âge supérieur ou égal à 38400 (Gif 4447) dans une niche de l'entrée de la grotte et de $43000 \pm 2400$ (Lyon 6961) dans la salle des lemmings. Un lambeau de cette couche moyenne a également été préservé de l'extraction de matériaux en 1846 et de l'érosion car il se trouve, à l'extérieur de la grotte, protégé au fond d'une petite niche relativement profonde. Un prélèvement effectué pour une datation par la méthode OSL a permis de dégager un os en cours de datation et un éclat de taille de très bon silex (ce n'est pas le silex local qui est gélif) et très frais. Ce dernier élément montre que ce lambeau de couche moyenne provient de la base de cette couche puisqu'il a incorporé cet éclat issu de la couche inférieure qui contenait l'industrie moustérienne. La datation ${ }^{14} \mathrm{C}$ de l'os ne nous est pas encore connue mais la méthode OSL

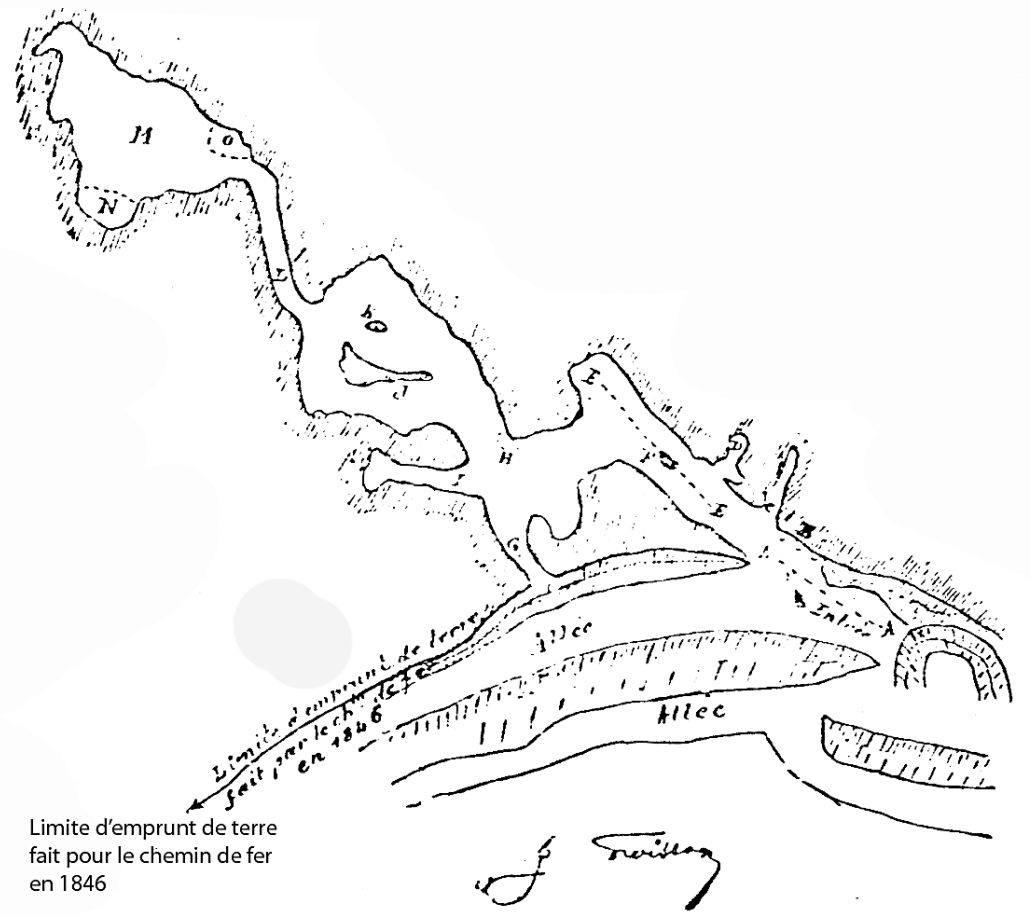

Figure 3 - Plan de la grotte F. d'Achon, levé en 1912 par l'architecte J. Froisson. Le plan indique clairement la limite de l'emprunt de terre qui a été fait pour la ligne de chemin de fer ToursAngers.

Figure 3 - Map of cave F. d'Achon, drawn in 1912 by architect J. Froisson. The plan indicates clearly the limit of taken sediments extracted for the railway between Tours and Angers. 


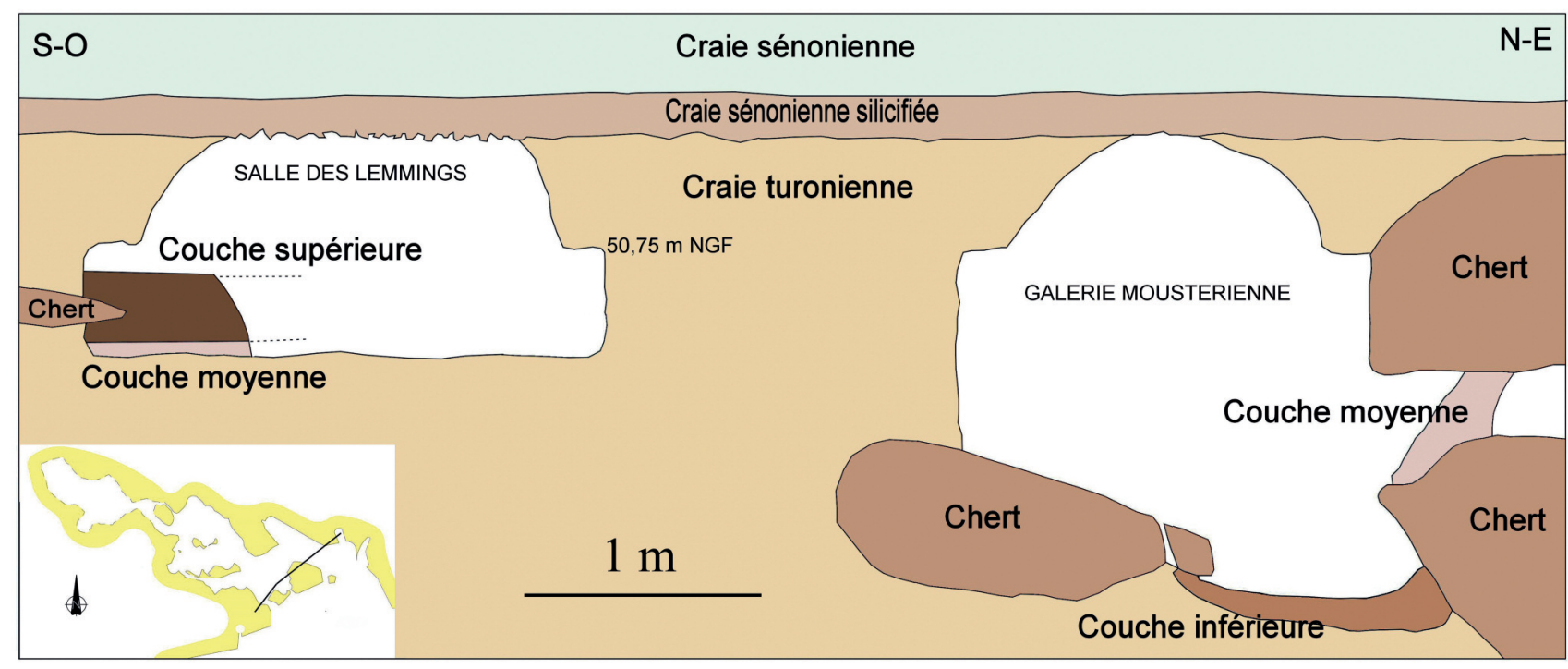

Figure 4 - La Roche-Cotard I. Stratigraphie des lambeaux de couches archéologiques.

Figure 4 - La Roche-Cotard I. Stratigraphy of bits of archaeological strata.

donne un âge de $72600 \pm 6000$ ans (MFGI Budapest) pour le sédiment de cette niche ;

- une couche supérieure préservée dans un très important témoin qui remplit complètement un conduit faisant communiquer la salle des lemmings avec l'extérieur. Une partie est toujours en place et recouvre un lambeau de la couche moyenne ; cet ensemble sera conservé. La nature de cette couche est foncièrement différente de celle de la couche moyenne, très riche en éléments cryoclastiques ; elle contient une faune qui confirme le caractère particulièrement froid de l'épisode climatique de sa mise en place. Un os a été daté de 34795 à 35942 av. J.-C. (Lyon 6962) par le ${ }^{14} \mathrm{C}$ et le sédiment de $64100 \pm 5100$ (MFGI Budapest) par la méthode OSL. Cette différence entre des dates obtenues par les deux méthodes tient au fait que pour la quasi-totalité des datations obtenues par le ${ }^{14} \mathrm{C}$ à La Roche-Cotard, nous sommes proches de la limite de validité de la méthode ;

À côté de ces lambeaux se trouvant près de l'extérieur de la grotte, il en existait d'autres, plus éloignés de l'entrée : - une couche intacte de 7 à $8 \mathrm{~cm}$ d'épaisseur non atteinte par d'Achon dans la partie nord de la salle du pilier est constituée d'argile particulièrement compacte qui contenait quelques rares fragments d'os anguleux, des dents isolées mais aussi une grande quantité de petits fragments d'os spongieux. Un seul silex taillé a été découvert dans cette couche. Le sédiment est assez pauvre en grains de pollens. Une lame pour étude micromorphologique montre la grande compacité du sédiment et la microstructure confirme qu'un tassement important a affecté cette couche datée de $47500 \pm 3000$ par le ${ }^{14} \mathrm{C}$;
- dans le dernier espace accessible de la grotte, la salle de la hyène, un épais remplissage a été en partie fouillé par $F$. d'Achon qui y a collecté un nombre important de restes de grands mammifères brisés et rongés par les hyènes ainsi qu'un crâne presque complet de hyène. Une dent d'équidé, oubliée par d'Achon, a été découverte reposant sur la surface indurée qui termine le remplissage alors que le sondage des couches sous-jacentes n'a pas donné de mobilier. Cette dent a été datée de $44600 \pm 2300$ par le ${ }^{14} \mathrm{C}^{7}$.

\section{2 - La stratigraphie de La Roche-Cotard IV}

La réouverture du chantier en 2008 était notamment motivée par le souhait de poursuivre un ancien sondage ouvert audelà de La Roche-Cotard III, qui n'avait pas permis d'atteindre la paroi de craie tuffeau. Une tranchée d'un mètre de large puis une fouille dans une petite grotte-abri découverte à la base de la paroi ont permis de mettre en évidence une stratigraphie de dix mètres de puissance (fig. 5).

Les couches rencontrées sont le résultat d'apports divers certains latéraux externes dus à des débordements de la Loire (couches 10 et 11), d'autres latéraux internes dus à la dissolution des calcaires qui forment le substrat (couche 22) et, enfin, les derniers, verticaux, riches en plaquettes de gel (couches 7 à 9) ou essentiellement argilo-limonosableux (couches 2 à 6), issus du démantèlement de la paroi et des colluvionnements venus du plateau. La couche 22, la plus ancienne, a été datée de $189000 \pm 14900$ ans par la méthode OSL (MFGI Budapest). Les couches de la partie moyenne du remplissage, (c 12 et 13), ont livré quelques silex taillés, toujours d'une très grande fraîcheur et quelques os brisés, parfois brûlés, rarement travaillés

(7) Les dates avec 1 sigma aussi fort ont été notées : > 40 000, dans le tableau 1 


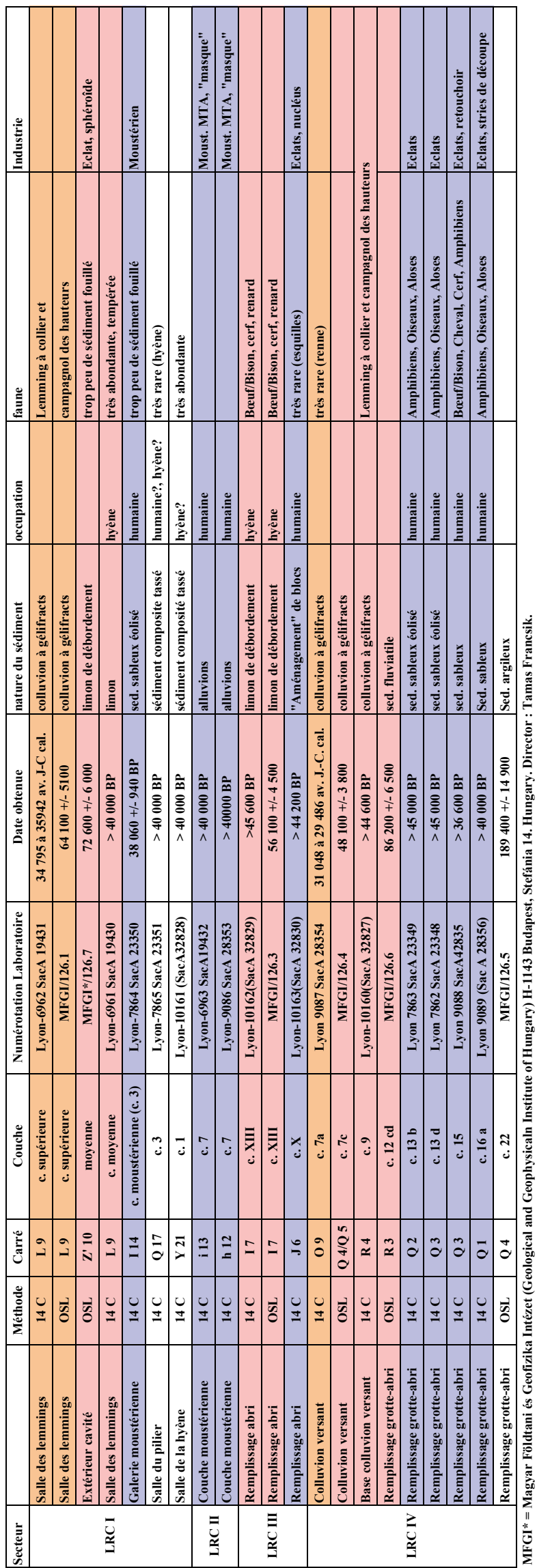

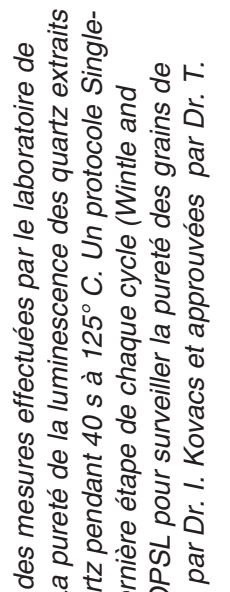

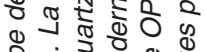

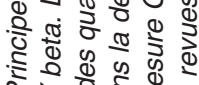
Q

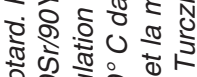

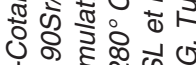

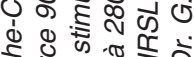

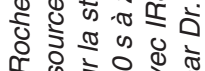

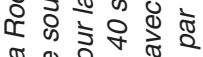

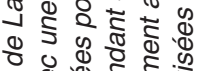

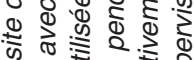
कि ริㅇํㅇำ

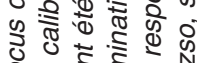
응 ธे है

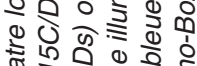

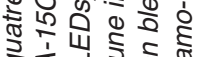

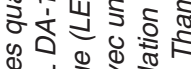

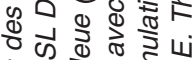

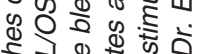

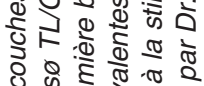

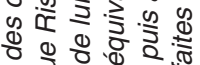
के

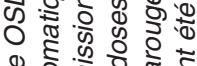

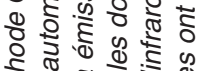
密 $-\infty$

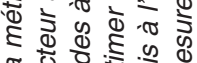

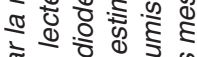
ब5 50 \% o. 0

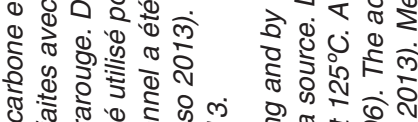

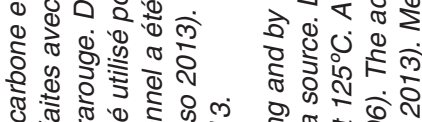

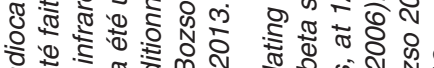

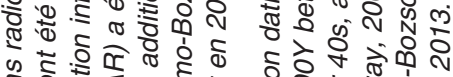
का व व 0 क क

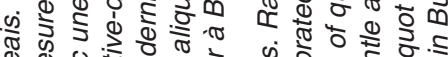

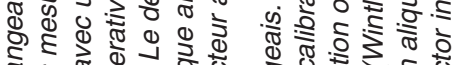

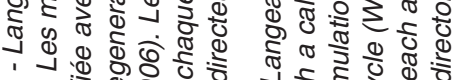

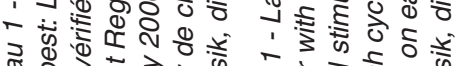

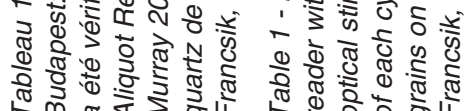


Nord

Q8

Q9

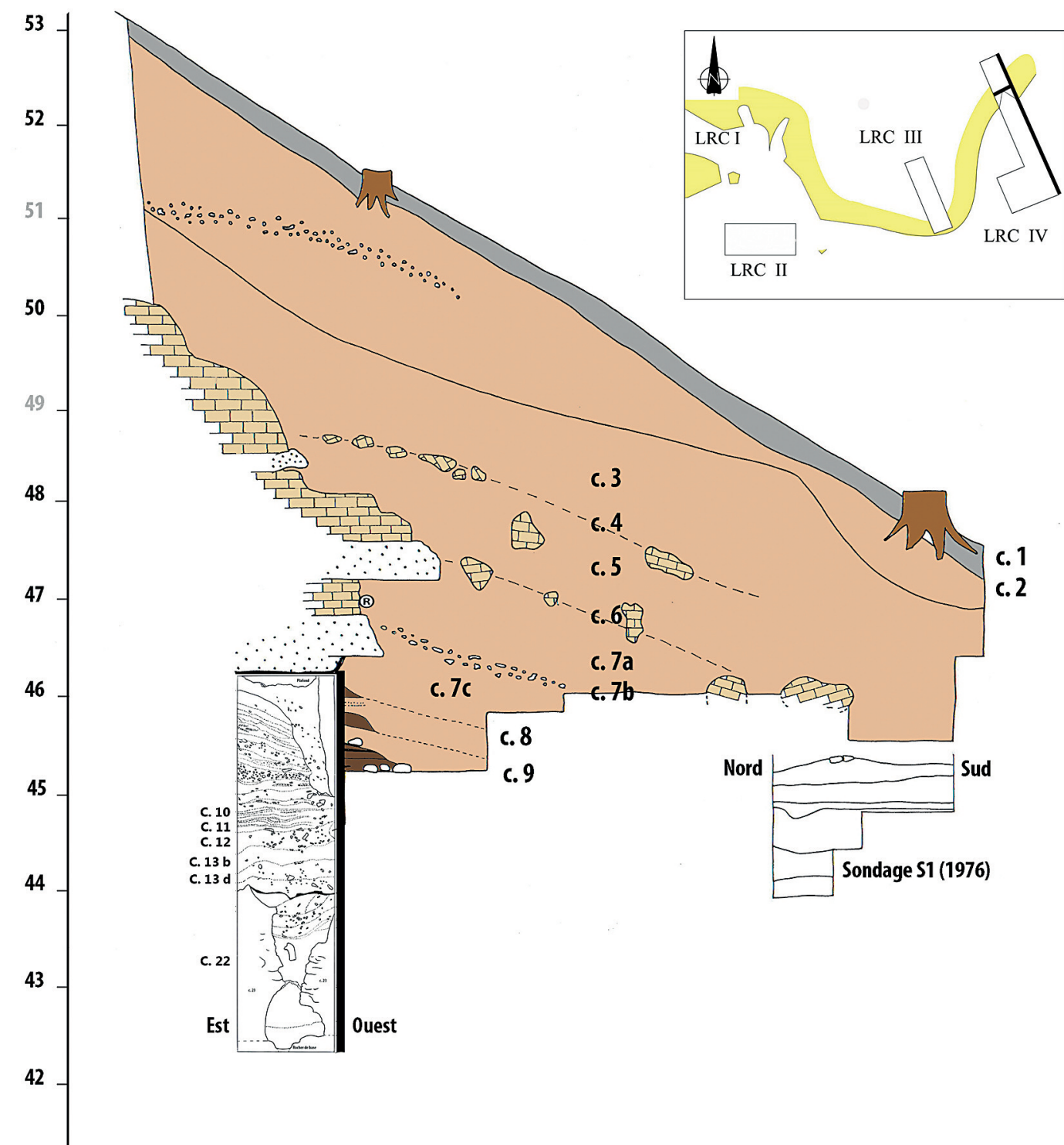

Figure 5 - La Roche-Cotard IV. Stratigraphie complète du locus. La partie supérieure est nord-sud, la partie inférieure est est-ouest.

Figure 5 - La Roche-Cotard IV. Total stratigraphy of the locus. The upper part is north-south, the lower part is east-west.

(incisions par silex, retouchoir). La couche 12 a été datée par la méthode OSL de $86200 \pm 6500$ (MFGI Budapest), les couches 13b et 13d d'un âge supérieur à 45000 (Lyon 7862 et Lyon 7863). Nous reviendrons plus loin sur l'intérêt que présente cet épais remplissage.

\section{3 - Contexte géologique, géométrie et géomorphologie du site}

Un contexte géologique très particulier est à l'origine de la formation de la cavité principale. Ce contexte tient en trois points (fig. 6) : la nature de la craie tuffeau jaune du Turonien supérieur, la présence au sein de la craie d'énormes masses siliceuses tenaces (nous utiliserons le terme : chert pour ce matériau presque impossible à tailler), enfin l'existence, à la base de l'étage Sénonien qui repose sur la craie tuffeau et constitue le plafond de la cavité, d'un niveau induré, solide sous la forme d'une calcarénite fortement silicifiée

Si l'extraction des matériaux en 1846 n'avait pas été effectuée, nous aurions ici, comme nous l'avons en amont et en aval, un rebord de plateau s'inclinant progressivement 
jusqu'à rejoindre la surface des alluvions modernes de la Loire, et un versant convexe dans sa partie supérieure et concave en bas. L'emprunt de terres a complètement modifié ce profil, le rendant très abrupt sur toute la longueur de l'emprunt avec des variantes résultant de la présence des énormes masses cherteuses au niveau des locus I et III où le décaissement de matériau n'a pas pu se rapprocher autant du rebord du plateau qu'à l'est et à l'ouest. L'étude d'impact réalisée à l'occasion du projet de viaduc de l'autoroute A-85, aujourd'hui construit pratiquement sur le site (Rialland et al. 1995) a révélé, grâce à des forages, l'existence de la falaise ennoyée et, au pied de celle-ci, à dix mètres de profondeur, un lambeau d'alluvion de la Loire à la même altitude que celui de LRC II. Le profil, suggéré par les sondages, encore réalisé dans la zone d'emprunt, vers son extrémité est, est tout à fait semblable à celui de LRC IV. II confirme la nature de ce versant dont les dépôts de pente masquent une falaise fossile ennoyée.

\section{4 - Les traces pariétales de la grotte d'Achon}

\section{1 - Le contexte géologique pariétal}

La cavité est creusée dans la craie tuffeau jaune du Turonien supérieur (fig. 6), naturellement tendre même en présence de la roche saine. À l'air, la craie subit une altération qui, à la longue, près de l'entrée de la grotte, par exemple, provoque son effritement et la chute d'une fine poudre au pied de la paroi. Cette mince couche de tuffeau altéré, en place, peut sans peine être entamée par l'extrémité d'un doigt sans exercer une forte pression.

Un très mince film brun argilo-limoneux, homogène, très tendre lui aussi, de quelques millimètres d'épaisseur maximum, a recouvert la paroi, en en épousant parfaitement la forme (fig. 7). À l'origine, il devait recouvrir complètement la paroi, aujourd'hui, il manque localement pour des raisons diverses : griffades des animaux cavernicoles, (le matériau reste présent dans les parties concaves protégées de la paroi), traces de doigts ou d'instruments divers (un raclage porte un graffiti récent) et par l'effet de la pesanteur lorsqu'il s'agit de très minces placages (très localisés) sur la paroi, etc. Ce film argilo-limoneux est aujourd'hui encore d'une très grande fragilité.

Son analyse des argiles par diffractométrie $\mathrm{X}$ du matériau, prélevé dans la salle de la hyène, montre qu'il ne peut pas provenir de l'altération in situ de la craie tuffeau (Touron, Bourgès 2012). Son épaisseur n'est d'ailleurs pas constante: en hauteur, elle peut être épaisse de 3 à $4 \mathrm{~mm}$ ou bien former des grumeaux qui sont restés accrochés au tuffeau.

\section{2 - Les panneaux de tracés de La Roche-Cotard}

\subsection{1 - Vue d'ensemble de la paroi nord de la salle du pilier}

La paroi nord de la salle du pilier a fait l'objet d'une couverture photographique par Y. Egels (objectif de $18 \mathrm{~mm}$, détails en $55 \mathrm{~mm}$ ). Les clichés permettent d'obtenir une représentation en 3D de cette partie de la salle (fig. 8). Une première exploitation a été réalisée avec le logiciel 123D Catch.

Les principaux caractères de la paroi apparaissent bien sur la photo :

- le plafond constitué par la base du Sénonien montre une très grande hétérogénéité ;

- la forme de la paroi de craie tuffeau du Turonien supérieur atteste de la texture ici très homogène, constante de la craie, si l'on fait abstraction des masses cherteuses, depuis l'entrée de la grotte jusqu'à la salle de la hyène. Cette paroi porte des stigmates de l'histoire de la cavité ;

- au-dessus de la ligne g, la paroi de tuffeau est en partie recouverte d'une mince pellicule (3 à $4 \mathrm{~mm}$ au maximum) d'un matériau limono-argileux brun rougeâtre ;

- à la base de la coupe, le niveau horizontal formé par les dalles de chert est bien visible ;

- sous la dalle de chert et la couche d'argile de décalcification qui l'accompagne, on retrouve la craie tuffeau.

\subsection{2 - Les quatre principaux panneaux de tracés digitaux}

Sur les parois de la cavité où le limon est encore présent se trouvent de très nombreuses traces d'origine anthropique. Nous nous limiterons ici à présenter les quatre principaux panneaux, les plus faciles à lire et à relever.

\subsubsection{1 - Le panneau à représentation circulaire}

Ce panneau complexe, non visible sur la figure 8 , se trouve un peu plus à droite, sur la même paroi. Nous n'en décrirons que la figuration la plus apparente. Elle est associée à une autre figure plus difficile à lire et qui apparaît sur une plage de paroi qui semble avoir été débarrassée intentionnellement d'une grande partie de son revêtement limoneux superficiel.

La surface sur laquelle a été tracée cette figure de $18 \mathrm{~cm}$ au carré (fig. 9) possède un revêtement limoneux qui peut localement atteindre 3 ou $4 \mathrm{~mm}$. Au fond du tracé, la couleur du matériau est très différente de celle du limon, il s'agit alors de la pellicule superficielle de craie tuffeau qui a été altérée. Elle est restée en place, piégée sous le limon. La structure circulaire est composée, à gauche, de deux tracés sub-parallèles et, à droite, de quatre tracés exécutés en même temps, de la même main. Les deux tracés à gauche se terminent, en bas, par deux impacts circulaires, probablement de doigts, identiques à quatre ou cinq autres impacts qui se trouvent en bas à gauche de la figure circulaire. De la même manière, le trait subhorizontal de la base de la figuration semble issu d'un impact très proche mais nettement séparé de l'un des deux impacts de gauche. Les quatre tracés «parallèles» situés à droite ne sont pas liés à des appuis ni à une extrémité ni à l'autre. À priori, le parcours des quatre doigts est descendant. Logiquement, les deux tracés de gauche devraient être descendants et se terminer par un fort impact comme le tracé inférieur qui démarre avec une trace du même type. L'essai d'interprétation technique (fig. 10) propose, pour chaque trait, le sens dans lequel il aurait pu être réalisé. L'hésitation est possible en ce qui concerne les deux traces incurvées qui sont à gauche. À l'une de leurs deux 


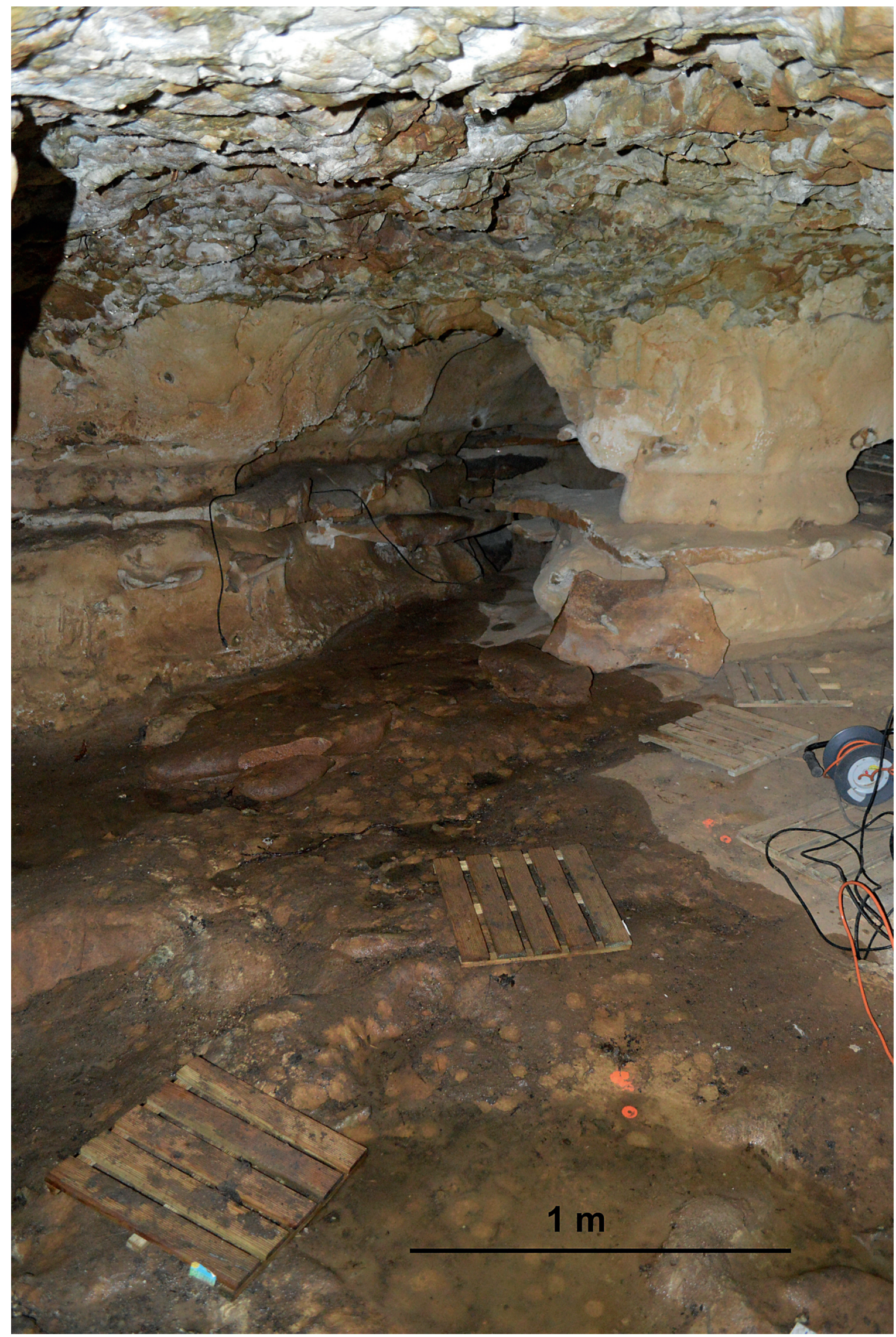

Figure 6 - La Roche-Cotard I. Le plafond de la cavité est constitué par une biocalcarénite fortement silicifiée dont le cortège fossile indique le Sénonien. Les parois montrent la craie tuffeau tendre du Turonien supérieur. Des bancs de chert sont visibles au sein du Turonien.

Figure 6 - La Roche-Cotard I. The ceiling of the cave is made of a strongly silicified biocalcarenitewhose fossils indicate Senonian. The walls show soft «tuffeau chalk» of upper Turonian. Levels of «chert» are visible inside Turonian. 


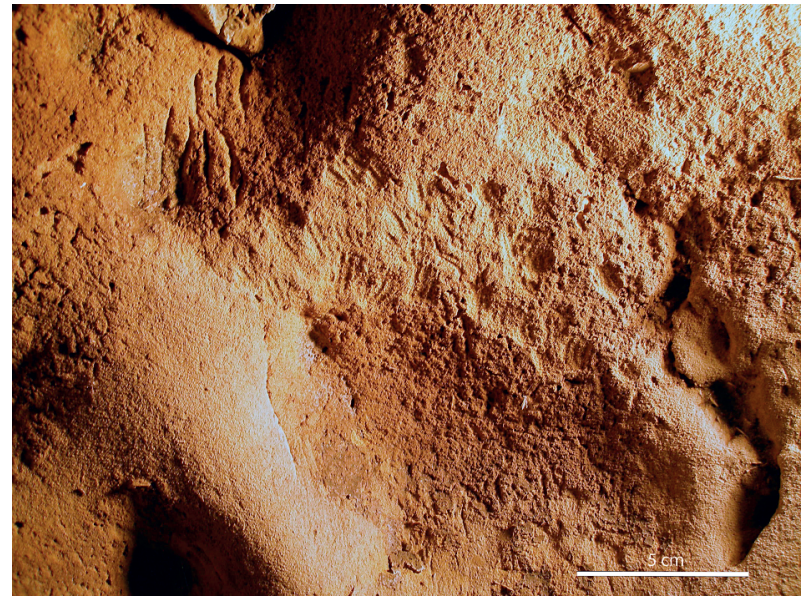

Figure 7 - La Roche-Cotard I. Dans la salle du pilier, la paroi est recouverte d'un film argilo-limoneux, absent sur les parties en relief et entamé par de nombreuses griffures d'animaux.

Figure 7 - La Roche-Cotard I. In " la salle du pilier ", the wall is covered with a silty clay which is no present on raised relief and cut in by numerous animal grazings. extrémités, on observe un large impact punctiforme qui semble dû à un appui insistant du doigt sur le limon. II paraîtrait plus logique de voir cet appui au démarrage du tracé mais ce serait alors un tracé remontant, ce qui ne semble pas bien correspondre à un mouvement naturel de la main et du bras. L'alternative est que cet appui fort termine le tracé et, dans ce cas, le mouvement qui aboutit au tracé, du haut vers le bas, est un mouvement naturel facile à réaliser. Une analyse ultérieure de macrophotos pourra peut-être permettre de trancher entre les deux possibilités.

\subsubsection{2 - Le panneau triangulaire}

Ce panneau est visible sur la figure 8 en $d$. II a sensiblement la forme d'un triangle isocèle (fig.11) dont la base, située en haut, mesure $44 \mathrm{~cm}$. La hauteur, axe de symétrie du triangle, mesurée jusqu'au centre du petit rognon cherteux qui se trouve au sommet opposé à la base, est de $30 \mathrm{~cm}$. Ce panneau se trouve en haut de la paroi de craie tuffeau immédiatement au-dessous du plafond. Le côté du triangle isocèle, à gauche, est bien marqué par un abrupt de quelques centimètres de hauteur ;

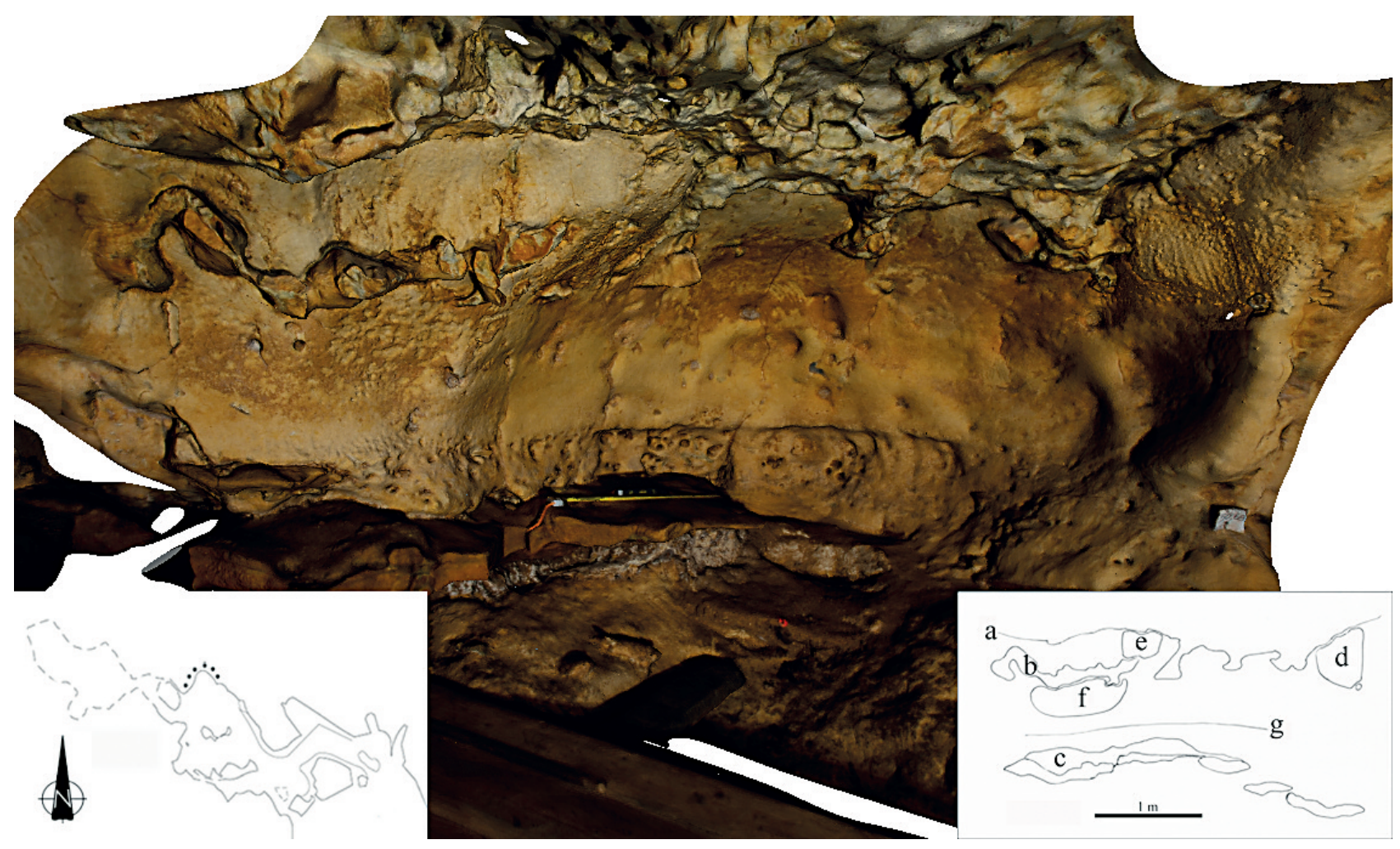

Figure 8 - La Roche-Cotard I. La salle du pilier. Vue 3D de l'alcôve dont la paroi porte les panneaux de tracés (photo Y. Egels). A gauche, la localisation de cette alcôve dans la salle. A droite, la localisation des différents panneaux. a. Limite du plafond sénonien et de la paroi turonienne, $b$. Chert, $c$. Dalle de chert inférieure, $d$. panneau de tracés triangulaire, e. Panneau de tracés rectangulaire, f. Panneau de ponctuations, $g$. Limite supérieure de la paroi soumise à l'action dissolvante de l'eau lors d'un épisode antérieur à l'occupation de la grotte par l'homme.

Figure 8 - La Roche-Cotard I. La salle du pilier. 3D view of the alcove whose wall has markings panels(photo Y. Egels). On left, the situation of that alcove in the room. On right, the position of the different panels. a. Limit between senonian cieling and turonian wall. b. "Chert». c. Lower "chert» level. d. Triangular panel. e. Rectangular panel. f. Punctuation panel. g. Upper limit of the wall which has been dissolved during a phase before occupation of the cave by man. 


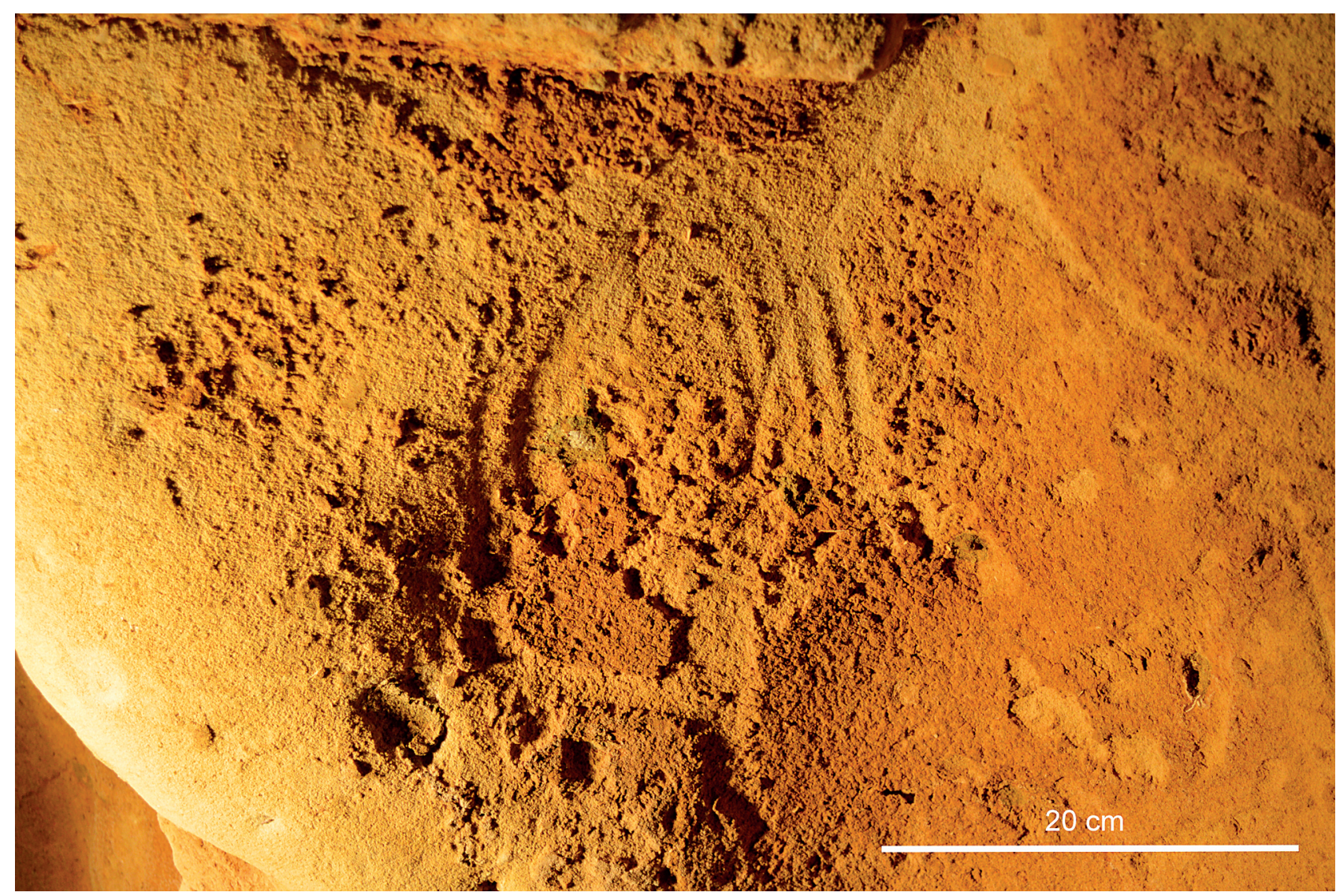

Figure 9 - La Roche-Cotard I. Panneau à représentation circulaire dans la salle du pilier.

Figure 9 - La Roche-Cotard I. Panel with circular form in «la salle du pilier».

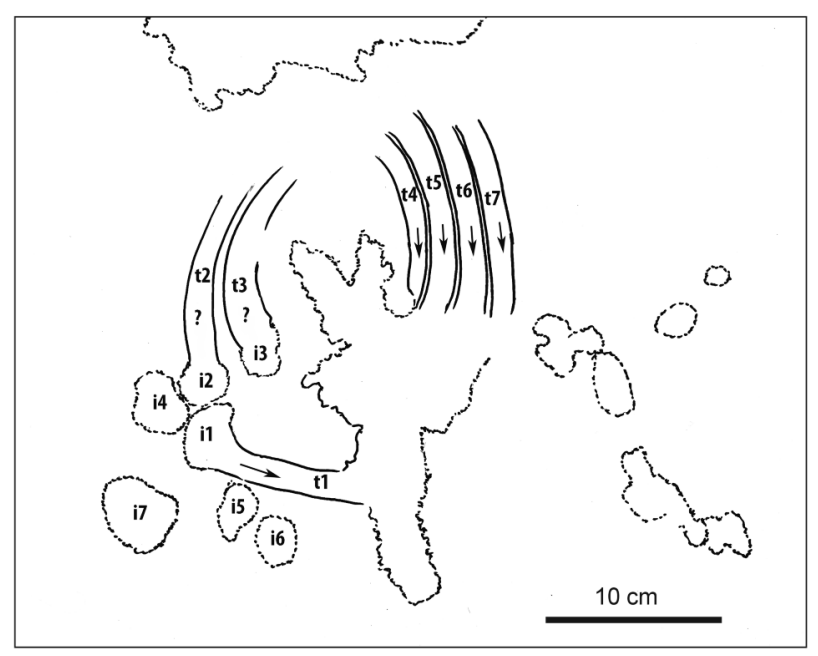

Figure 10 - La Roche-Cotard I. Essai d'interprétation des tracés du panneau à représentation circulaire dans la salle du pilier.

Figure 10 - La Roche-Cotard I Interpretative essay of the panel with circular form in " la salle du pilier ». l'autre petit côté coïncide avec une sorte d'avancée de la paroi, bourrelet presque vertical dans sa moitié supérieure, nettement plus oblique, vers la gauche, dans sa moitié inférieure. Au sommet du triangle, sur son axe de symétrie, le petit chert de section légèrement ovale, est en position naturelle ; il émerge du tuffeau, et sa section mesure $45 \mathrm{~mm}$ de longueur et $30 \mathrm{~mm}$ de largeur.

Le panneau triangulaire est couvert de tracés digitaux ou réalisés à l'aide d'un instrument ayant la forme et la taille d'un doigt (nous parlerons alors de tracés digitaux), sensiblement parallèles, perpendiculaires à la base du triangle. Ces tracés sont un peu moins visibles sur la partie droite que sur les parties gauche et centrale. Ils préservent, dans le panneau, sur le petit côté à gauche, trois triangles de limon qui prolongent le film de sédiment encore en place au-dessous du panneau (fig. 12). Le limon du triangle le plus à gauche, le plus grand, a une épaisseur de l'ordre de 4 à $5 \mathrm{~mm}$ mais cette épaisseur comprend peut-être également une partie de la pellicule de tuffeau altéré qui est piégée dessous. Le triangle suivant qui est situé à peu près au milieu du côté du triangle est formé d'un limon dont l'épaisseur est de l'ordre de $3 \mathrm{~mm}$. Enfin, un troisième triangle est visible plus à droite ; l'épaisseur du limon y est plus faible que sur les deux précédents et il est également plus petit. 


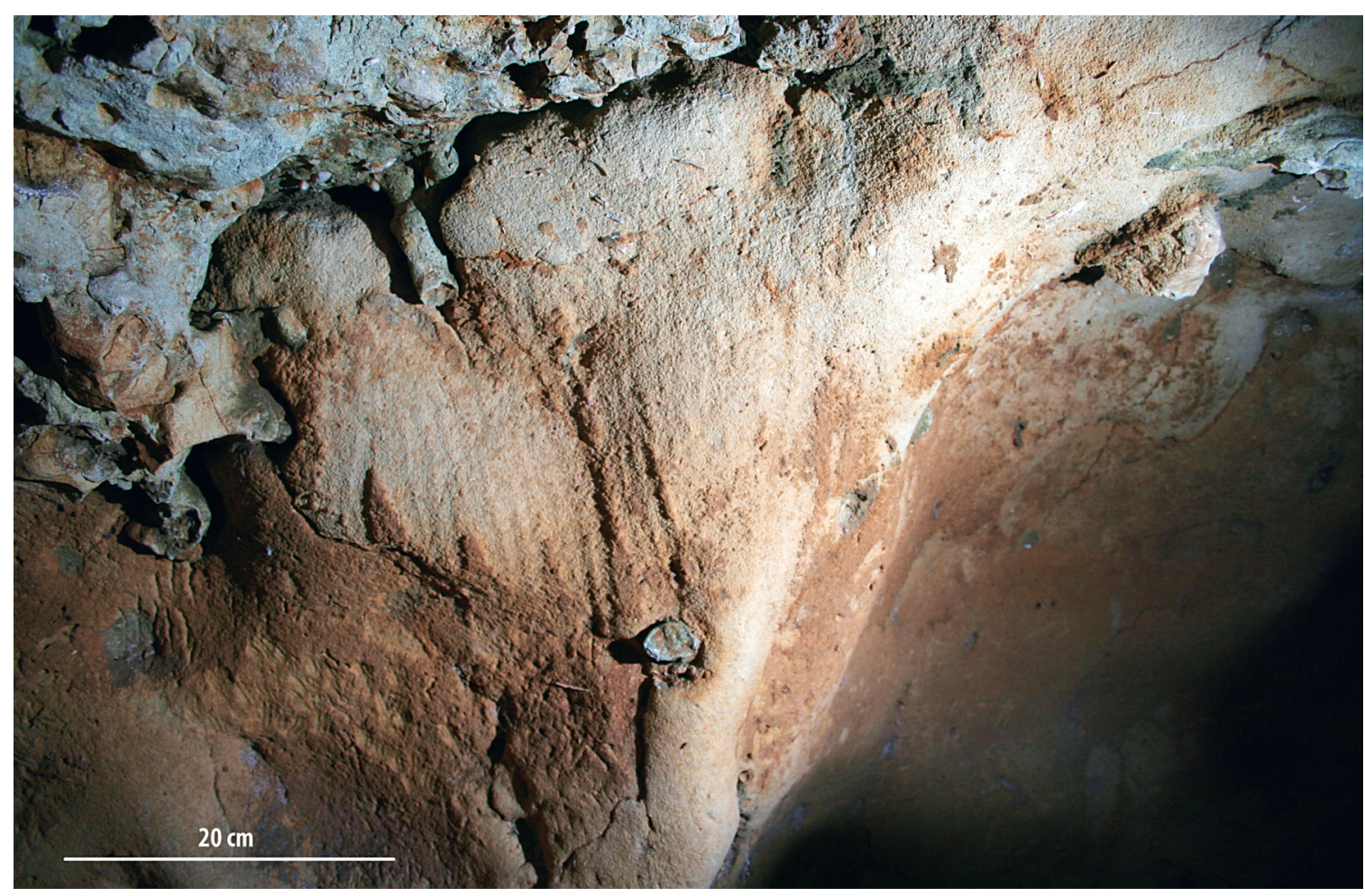

Figure 11 - La Roche-Cotard I. Panneau de tracés triangulaire (fig. 8d).

Figure 11 - La Roche-Cotard I. Triangular panel (fig. 8d).

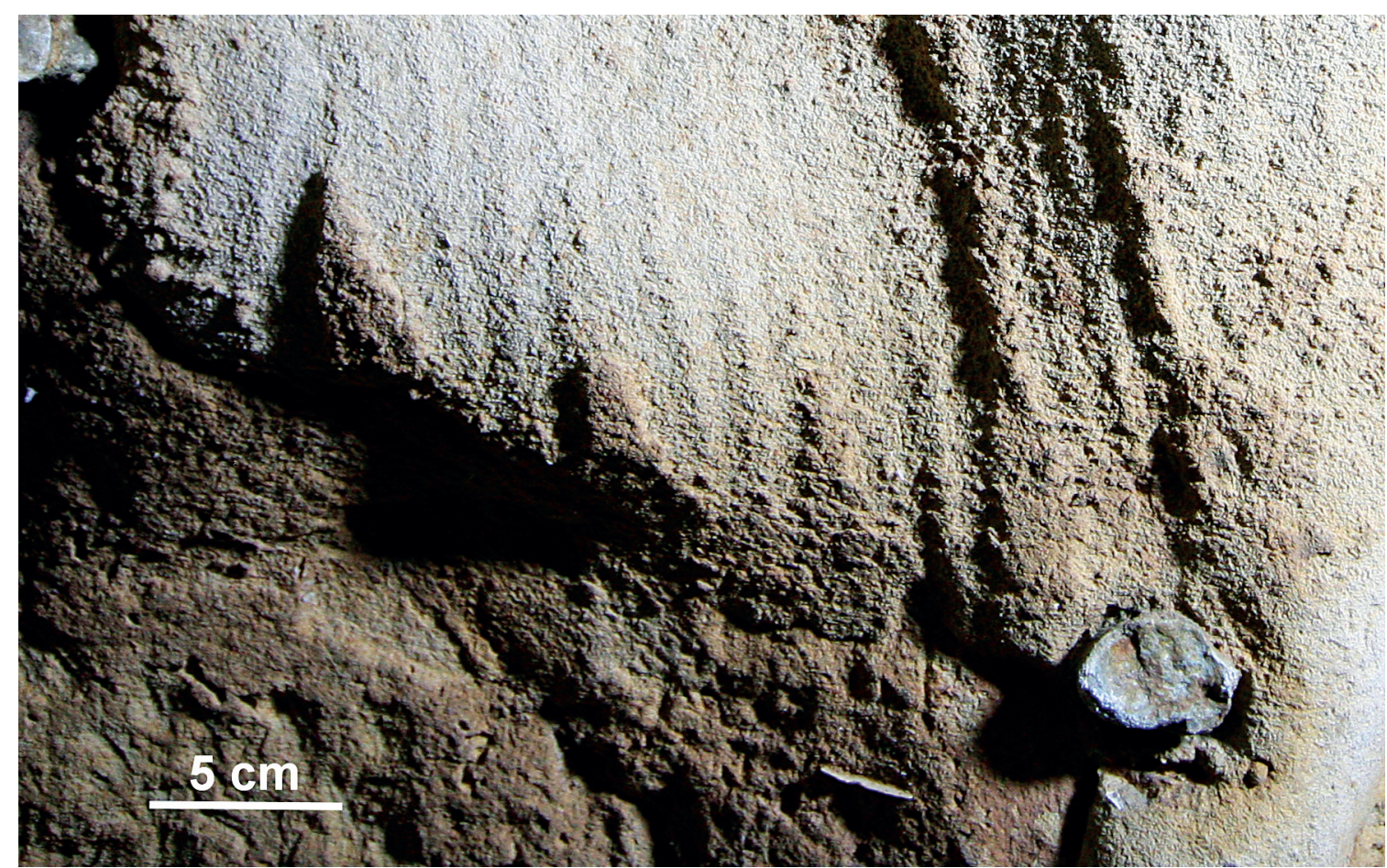

Figure 12 - La Roche-Cotard I. Détail du panneau de tracés triangulaire montrant les zones réservées (trois triangles et deux étroites bandes parallèles.

Figure 12 - La Roche-Cotard I. Triangular panel. Detail showing reserved parts (three triangular parts and two narrow parallel lines). 
La photogrammétrie et son traitement en image colorée, dus à Y. Egels (fig. 13) indiquent une équidistance entre les courbes de l'ordre de $1 \mathrm{~mm}$. Le relief est bien perçu au niveau des triangles, notamment sur le plus à gauche, où l'on peut remarquer la très nette dissymétrie entre les deux versants principaux de ce triangle de limon préservé. Les parties en creux apparaissent en orange et rouge, les parties en relief apparaissent en vert et bleu. La forme des deux «synclinaux» qui encadrent le grand triangle suggère que l'opérateur s'est appliqué dans la réservation de cette zone.

Le relevé interprétatif (fig. 14) montre deux grandes arêtes parallèles axiales ( $d$ et e) qui séparent le panneau en trois parties : la partie gauche possède les trois triangles de limon résiduel $(\mathrm{a}, \mathrm{b}$ et $\mathrm{c})$; une partie centrale rectangulaire formant une bande axiale se prolonge vers le bas, par le rognon cherteux à section ovalaire ; enfin, la partie droite montre encore des tracés digitaux un peu moins lisibles que les autres mais un éclairement bien orienté permet d'observer ces traces jusqu'à l'extrémité de la partie droite du triangle.

L'examen de la partie gauche du panneau révèle un certain rythme dans la succession des tracés digitaux et des trois triangles résiduels (fig. 14) : complètement à gauche, trois tracés digitaux parallèles $(1,2$ et 3$)$ passent à gauche du grand triangle de limon (a) ; ensuite un ensemble de trois tracés digitaux (4, 5 et 6$)$ aboutissent entre le triangle précédent (a) et le suivant (b) ; entre b et $c$, un nouvel ensemble de trois tracés $(7,8$ et 9$)$; enfin, pour cette première partie du panneau, un ensemble de trois tracés parallèles $(10,11$ et 12$)$ est placé entre ce dernier triangle et la première des deux arêtes axiales. Celles-ci (d et e) sont très nettement séparées par un ensemble de trois tracés digitaux $(13,14,15)$ rigoureusement parallèles. Elles forment des reliefs nettement plus accusés que ceux qui séparent les tracés digitaux contigus précédents. En section, leur largeur et leur hauteur sont de l'ordre de 4 à 5 $\mathrm{mm}$ dans leur partie médiane. Ces deux arêtes se terminent vers le bas par deux parties plus larges où une plus grande quantité de limon a été préservée. La bande formée par les deux arêtes et les trois tracés qui les séparent très nettement, constituent une sorte d'axe de symétrie du triangle isocèle. À droite de la seconde arête (e), les tracés digitaux sont moins visibles et on ne retrouve pas les mêmes zones triangulaires réservées comme dans la partie opposée. On peut cependant dénombrer 8 ou 9 tracés à droite de l'arête $(16,17$, etc.) puis deux longs tracés qui semblent descendre au moins jusqu'au niveau du petit chert à section ovalaire. D'une manière générale, i ne semble pas possible d'émettre des hypothèses à propos de l'ordre dans lequel ont été effectués ces différents tracés. En revanche, même si aucune observation ne permet de le justifier, on ne voit pas comment ces tracés auraient pu être réalisés autrement que du haut vers le bas. Plus à droite, le placage de limon reprend, localement granuleux, il présente des manques.

\subsubsection{3 - Le panneau rectangulaire}

Ce panneau correspond à la plage e de la figure 8 . De forme sensiblement rectangulaire, il termine, vers la droite, une étroite bande horizontale de paroi de craie tuffeau qui se trouve entre le plafond de la salle et un assez long banc cherteux grossièrement horizontal (au-dessous). Comme pour le panneau précédent, il existe un vide au niveau du contact de la paroi turonienne et du plafond sénonien. Sa localisation, immédiatement sous ce contact, peut expliquer, comme pour le panneau précédent, sa bonne conservation, en hauteur, protégé du passage des animaux à fourrure (fig. 15). En bas à droite, une partie du panneau est vierge de traces de doigt (ou de l'instrument en forme de spatule qui aurait pu également être utilisé). En bas, à gauche, les traces sont stoppées par un abrupt ou le début du banc cherteux.

L'ensemble a une longueur de $35 \mathrm{~cm}$ et une hauteur de 25 $\mathrm{cm}$ en moyenne. II n'est pas vertical mais légèrement surplombant, son plan moyen faisant un angle de l'ordre de $15^{\circ}$ avec la verticale.

Le panneau montre de 27 à 30 traces à section concave, sensiblement parallèles, presque perpendiculaires au niveau du grand côté, formant un léger éventail ouvert vers le haut. II présente cependant quelques irrégularités dans les tracés avec des bifurcations dont trois sont orientées vers le bas et deux ou trois en sens inverse. Toutes les traces sont sensiblement de la même profondeur réduite. On peut se demander s'il ne s'agit là que de la partie profonde de ces tracés, la partie superficielle ayant pu être enlevée par érosion douce.

II n'y a pas de limon brun sur la composition : il est donc impossible d'affirmer qu'il en a été couvert comme pour les deux panneaux précédents. En effet, les lambeaux du matériau brun rougeâtre encore présent de part et d'autre du panneau ont pu être mis en place par un autre processus que par celui qui a permis le large recouvrement des zones plus basses. On peut cependant noter qu'il est à la même altitude que le panneau triangulaire et que logiquement, si cette pellicule de limon s'est bien mise en place grâce à un double phénomène de capillarité et dessèchement, elle devrait également avoir existé à l'emplacement de ce panneau.

\subsubsection{4 - Le panneau à ponctuations}

II est représenté en $\mathrm{f}$ dans la figure 8 . II se trouve sous le banc de chert qui nous a servi à situer le panneau rectangulaire (fig. 16). II appartient, comme les deux premiers, à une paroi couverte par le film brun. Dans ce secteur, le limon a recouvert toute la surface pariétale mais, dans sa partie basse, il a été enlevé très probablement à cause de frottements répétés. En dessous, la bande de film effacé est assez étroite et s'élargit d'une manière importante sur la droite. La limite entre la zone chargée du film de limon brun, en haut, et celle, en bas, qui en est dépourvue est très nette car la couleur brune tranche nettement sur celle du tuffeau. Néanmoins, la transition est progressive comme si le frottement avait été progressivement plus doux vers le haut. Partout où le film limoneux est présent, on observe des ponctuations de forme grossièrement circulaire d'un diamètre moyen de $2 \mathrm{~cm}$. Le fond des ponctuations montre la craie tuffeau pratiquement plane partout : il n'y a pas d'impact suffisamment fort pour entamer légèrement le 


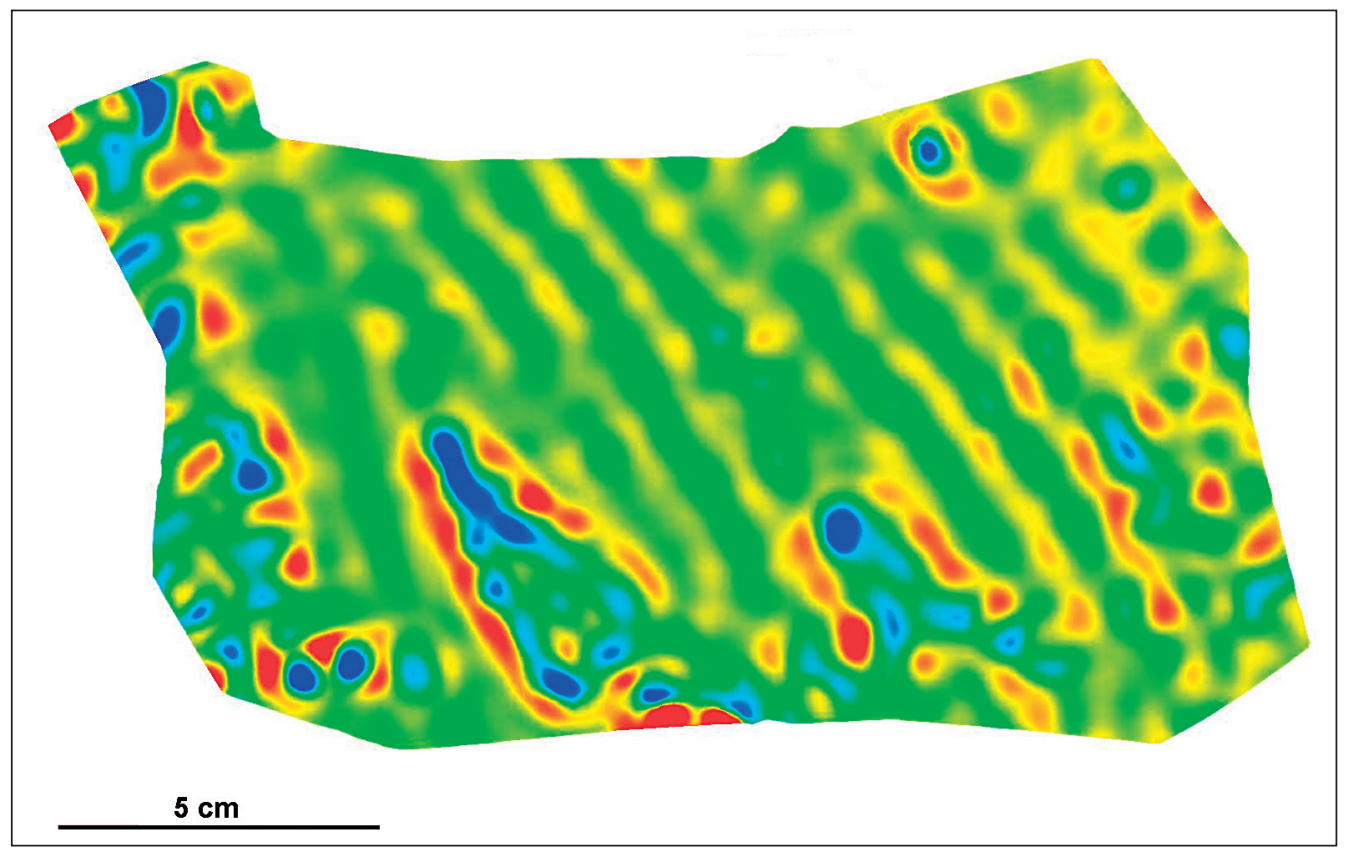

Figure 13 - La RocheCotard I. Traitement coloré d'une photogrammétrie de la même partie du panneau de tracés triangulaire. (Photo et traitement $Y$. Egels). Les parties orange et rouges sont «synclinales», les parties vertes et bleues sont " anticlinales ».

Figure 13 - La RocheCotard I. Triangular panel. Detail. Photogrammetry.

Treatment in color (Photo \& treatment $Y$. Egels). Orange and red parts are "synclinal ", green and blue parts are " anticlinal » parts.

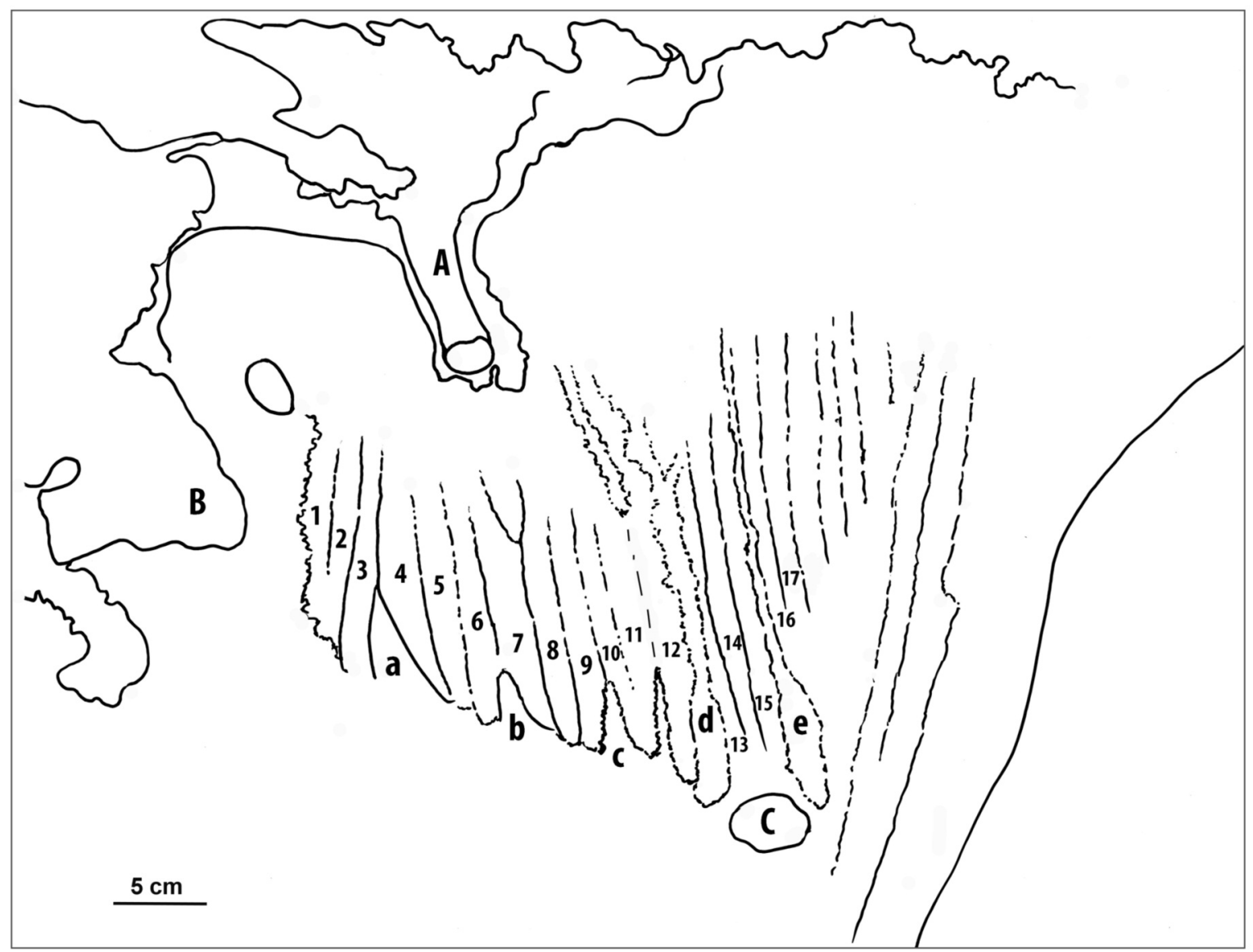

Figure 14 - La Roche-Cotard I. Relevé du panneau de tracés triangulaire.

Figure 14 - La Roche-Cotard I. Triangular panel. Interpretative essay. 


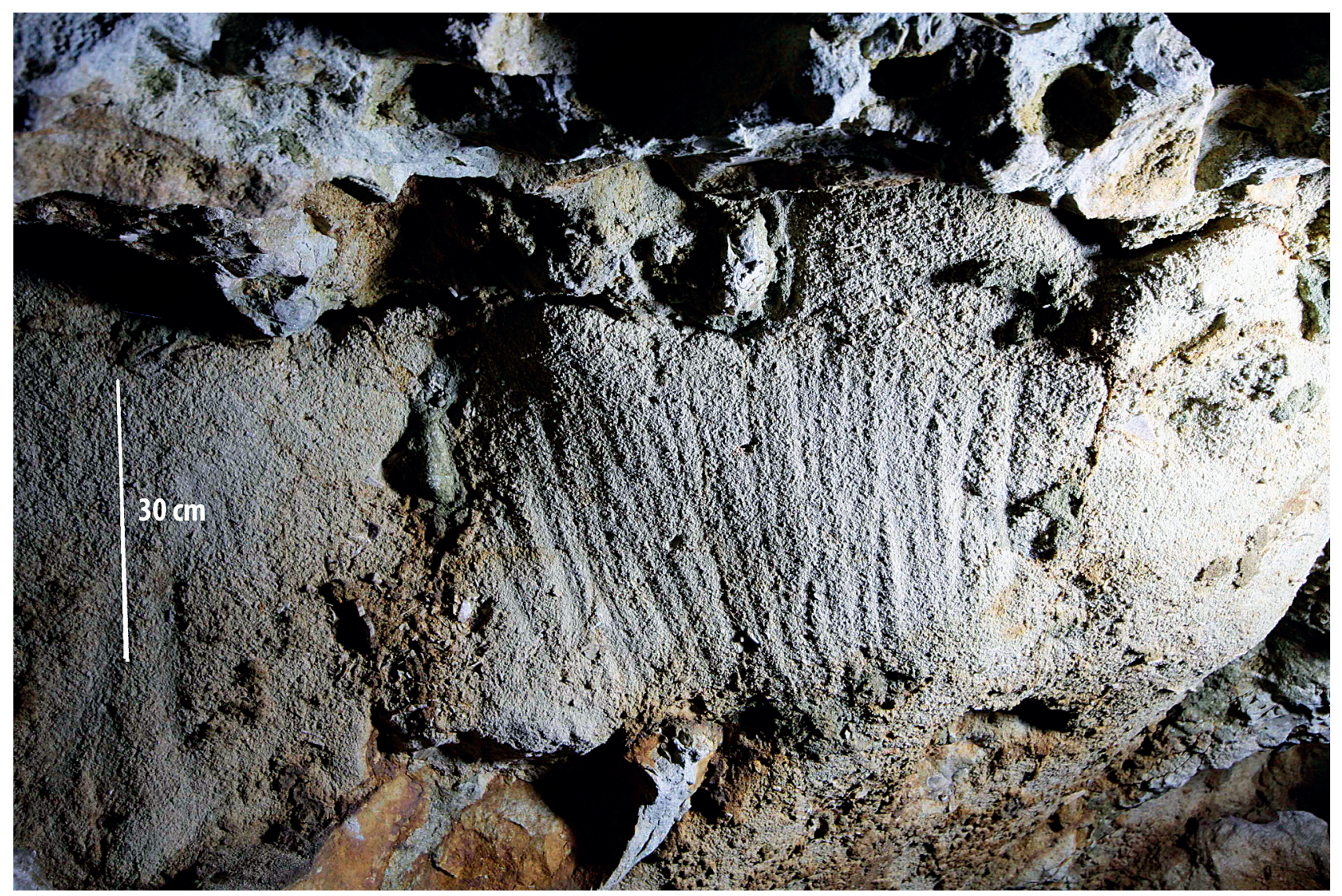

Figure 15 - La Roche-Cotard I. Panneau de tracés rectangulaire (fig. 8e).

Figure 15 - La Roche-Cotard I. Rectangular panel (fig. 8e).

tuffeau. Sous le chert supérieur, l'épaisseur de la couche limoneuse, de l'ordre de 3 à $4 \mathrm{~mm}$, est conservée jusque $25 \mathrm{~cm}$ plus bas ; elle décroît ensuite régulièrement jusqu'à disparaître complètement. Certaines ponctuations sont bien individualisées, bien séparées les unes des autres, surtout à gauche. En revanche, à droite, les ponctuations sont toujours parfaitement discernables, souvent jointives et peuvent même présenter une intersection (fig. 17). Vers le bas, au milieu, un groupe de sept à huit impacts semble récent.

\subsection{3 - Technique de réalisation des tracés}

Cet aspect de l'étude devra faire l'objet d'une recherche plus approfondie. Pour les trois panneaux, circulaire, triangulaire et rectangulaire, il semble évident que les traces ont été plutôt réalisées avec le doigt qu'avec un outil. Pour ce qui est du panneau à ponctuations, au contraire des précédents, les traces semblent avoir été réalisées avec un instrument plutôt qu'avec un doigt (le pouce ?) : en effet, la forme des ponctuations est grossièrement circulaire, mais certains impacts ont des formes dont une partie du contour est plus rectiligne. Par ailleurs, pour les traces allongées, nous avons fait, sur les schémas interprétatifs, des propositions de sens du mouvement

\subsection{4 - Altération des traces}

\subsubsection{1 - Le panneau triangulaire}

Sous ce dernier, la paroi forme un bourrelet vertical descendant, un autre se situe un peu plus à gauche. Ils déterminent une concavité dans laquelle le limon brun est conservé, portant des griffures fines et rapprochées (fig. 7). Sur les deux convexités, le film brun n'est pas conservé. Nous faisons d'ailleurs la même observation sur toutes les parties en relief de la moitié inférieure de la paroi de cette partie de la salle du pilier alors que, dans les creux, le limon est conservé. Cette érosion du revêtement limoneux est imputable aux passages répétés des moyens et/ou grands vertébrés qui ont fréquenté la cavité.

Par ailleurs, nous avons noté une moindre netteté des tracés digitaux sur la partie droite du panneau triangulaire. Ils ont donc subi une érosion mais qui ne peut être due aux passages des animaux. On peut d'ailleurs se demander si, dans cette salle, il n'y a pas eu à l'origine beaucoup plus de panneaux portant des tracés que ceux que l'on peut voir et décrire aujourd'hui. En particulier, sur la face nord du pilier qui lui a donné son nom, une autre large concavité fait face à celle de la figure 8 et sur laquelle, bien que dépourvue de 


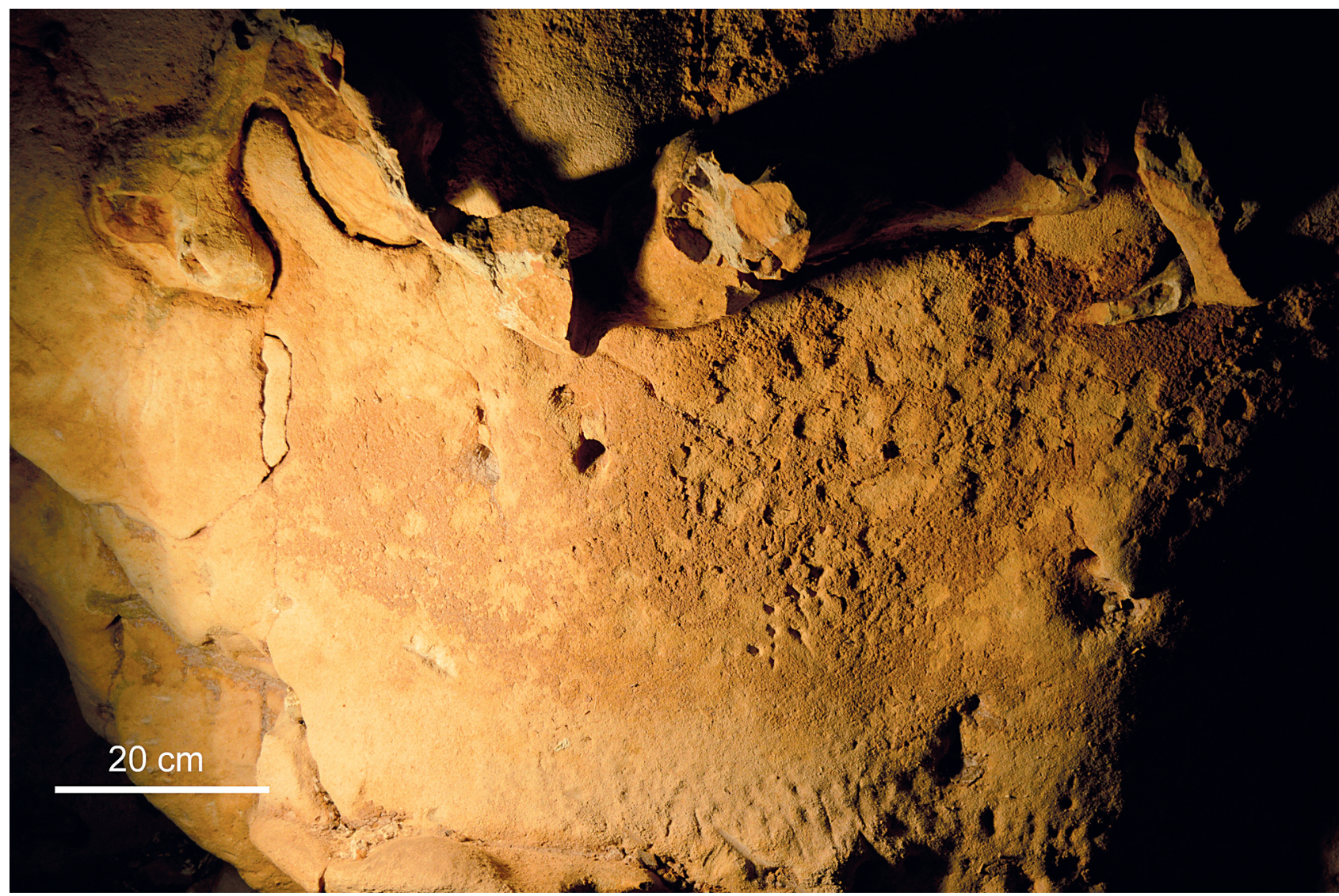

Figure 16 - La Roche-Cotard I. Panneau de ponctuations (fig. 8f).

Figure 16 - La Roche-Cotard I. Panel with punctuations (fig. 8f).

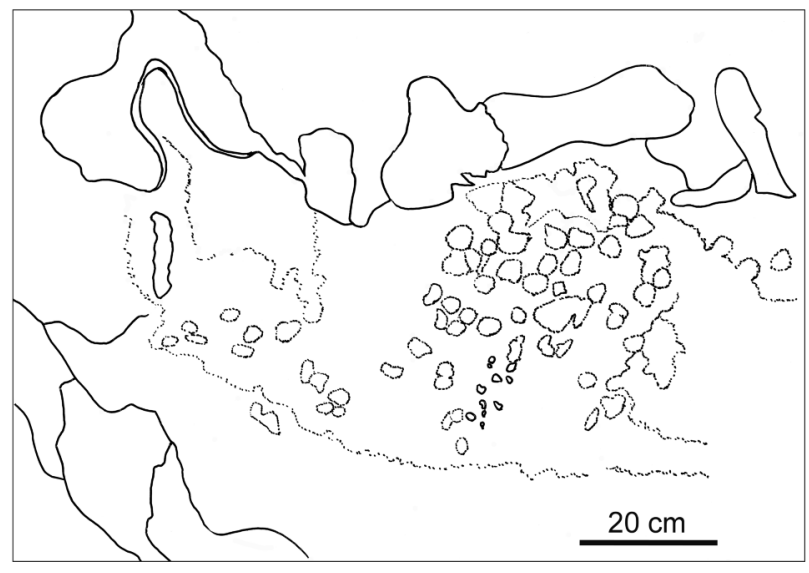

Figure 17 - La Roche-Cotard I. Relevé du panneau de ponctuations.

Figure17 - La Roche-Cotard I. Punctured panel. Plan. tout revêtement limoneux, des tracés digitaux très couvrants sont encore détectables.

Quant à la cause de l'érosion très douce qui a affecté certains tracés, une seule est possible : la circulation de l'air contre la paroi de la cavité. Si ces tracés ont bien 40000 ans ou plus comme nous en proposerons l'hypothèse, une telle durée paraît suffisante pour observer une érosion éolienne ainsi détectable.

\subsubsection{2 - Le panneau rectangulaire}

Ce panneau n'a pas conservé de lambeau de limon brun sur sa surface. L'explication paraît simple : les tracés parallèles sont tellement serrés que la totalité du revêtement brun a été enlevée au moment du traçage. Ces tracés ont également affecté la pellicule d'altération de la craie tuffeau puisqu'ils sont marqués dans l'épaisseur de cette pellicule aujourd'hui. La seule altération certaine est celle de l'altération superficielle, naturelle, de la craie.

\section{3 - Des taches de pigment rouge}

Au cours de leur mission de juillet 2009, P. Paillet et E. Man-Estier (Man-Estier, Paillet 2009) ont repéré dans le boyau qui conduit à la salle de la hyène à $280 \mathrm{~cm}$ de la sortie du boyau, plusieurs taches d'ocre sur la paroi 
(fig. 18). La première, bien visible est de forme sensiblement circulaire, mesurant quelques centimètres de diamètre. Le pigment a été déposé sur la surface dure d'un chert allongé de section cylindrique dégagé de la craie tuffeau par érosion différentielle. La deuxième tache, sur le même chert, à quelques centimètres de la première, est beaucoup moins visible et encore plus délavée. II existe enfin, mais cette fois à la surface de la craie tuffeau, une troisième trace de pigment rouge moins délavée. Elle présente une forme demi-circulaire, de $2 \mathrm{~cm}$ de diamètre environ. Elles demeurent très difficiles à observer dans la partie haute d'une petite niche qui se trouve au ras du sol, dans le boyau.

Il existe, dans le même boyau, mais sur l'autre face, côté sud-ouest, une quatrième tache ocre (fig. 19). Elle se trouve à deux mètres de l'entrée est du boyau, à proximité immédiate d'une large fissure que le phénomène de dissolution a élargie pour atteindre $5 \mathrm{~cm}$ de largeur et $18 \mathrm{~cm}$ de hauteur. De forme grossièrement circulaire, elle mesure un peu plus d'un centimètre de diamètre. Elle a probablement été en partie effacée du fait de passages répétés (traces d'arrachements horizontales à proximité immédiate).

\section{5 - De nombreux sites ont donné des tracés digitaux}

Si l'on définit les tracés digitaux comme un modelage réalisé au doigt sur une surface tendre (argileuse ou constituée de calcaire altéré type mondmilch) qui modifie l'aspect de cette surface sous l'effet d'un simple lissage en ruban (Lorblanchet 1995), plus de 65 sites présentant ce type de " décor » sont répertoriés dans le monde. Bednarik (1994) et Sharpe (2004) ont proposé des critères permettant de différencier l'origine de ces marques pariétales autres que celles anthropiques, réalisées avec le doigt ou un instrument quelconque. D'après les publications, les tracés digitaux se répartissent sur au moins 34 sites en Europe, dont 27 en France et 7 en Espagne, une trentaine en Australie (liste établie dans le cadre du «Parietal marking project» - Bednarik 1986, 2014), deux en Nouvelle-Guinée (Ballard 1992) et deux sur le continent américain : au Chili (Van Hoek 2011), en République Dominicaine (DuVall 2010).

Quant à la position chronologique, selon von Petzinger et Nowell (2011), des 27 sites français, les exemples se répartissent de l'Aurignacien au Magdalénien (datations basées sur des critères stylistiques et radiochronologiques). Un site de l'âge du Bronze découvert en 1989 (Aujoulat, Chevillot 1989) est en cours d'étude à la Grotte des Fraux (Petrognani et al. 2014).

Concernant les formes, figuratives ou non, la typologie proposée par Sharpe et Van Gelder (Sharpe, Van Gelder 2004, 2005) retient deux variables : à savoir si l'auteur/e a utilisé un ou plusieurs doigts pour réaliser un tracé, et si l'auteur/e était immobile ou pas en réalisant son tracé.

Pour une étude approfondie, il faudra comparer les tracés digitaux de La Roche-Cotard avec ceux d'autres sites contemporains ou apparentés par leur morphologie tels que Bara-Bahau, l'Aldène, Rouffignac, etc.

\section{6 - La datation de ces traces}

Comme précédemment noté (Lorblanchet 2008 ; ManEstier et al. ibid.), il ne fait pas de doute que ces tracés sont une œuvre humaine réalisée avec les doigts ou avec un outil dont l'extrémité serait très probablement de forme différente pour les tracés et pour les ponctuations. De même, les traces rouges du boyau ne peuvent pas être dues à la chauffe de la paroi par une flamme qui aurait donné une couleur différente allant vers le brun. Ici, le rouge est relativement vif et ne semble pouvoir être dû qu'à de l'ocre éventuellement mélangé avec une autre substance. L'âge de ces tracés? Nous ne pourrons obtenir une datation directe pour ces derniers. Un film blanc particulièrement mince existe à la surface de certains dépôts argileux naturels accrochés à la paroi. Seuls seront disponibles des éléments de datation indirects contextuels. Nous nous tournerons donc vers les résultats des datations par le ${ }^{14} \mathrm{C}$ et par la méthode OSL. Mais auparavant, nous montrerons que la géométrie des dépôts des quatre locus de La Roche-Cotard sont d'un apport déterminant pour ce qui est de l'approche de l'âge de l'occupation de la cavité par les hommes de Néandertal.

\section{1 - La géométrie des dépôts}

\subsection{1 - Les observations de F. d'Achon}

Dans son article (d'Achon ibid.) publié une année après la découverte de la grotte, F. d'Achon note : «...des emprunts considérables de terre ont été faits à cette partie du coteau de la Loire, entre Cinq-Mars-la-Pile et Langeais, à des époques différentes. Ces emprunts se rapportent d'abord au moment de l'endiguement de la Loire et surtout, en 1846, pour la construction du remblai du chemin de fer Tours-Nantes qui traverse, dans cette partie du parcours, les prairies de Langeais. La trace de ces derniers travaux se retrouve sur le rocher calcaire qui forme le flanc du coteau de la vallée où se distinguent encore les vestiges des coups de mine, de pique et de taille régulière de pierres.» F. d'Achon poursuit : «Ces ouvriers ont arrêté leur travail de déblai, dans le parc de La Roche-Cotard, audessous de l'entrée de la grotte et ont vraisemblablement fait disparaître là, comme ailleurs, de nombreux ossements et silex taillés qui sont alors passés inaperçus en raison $d u$ peu d'intérêt qu'on y attachait». L'inventeur de la grotte ajoute enfin à propos de la découverte : "L'entrée de la grotte était masquée par des éboulements et n'était accusée à l'extérieur que par une gueule de terrier. $A$ l'intérieur, derrière ce masque, elle était comblée jusqu'à 30,40 ou 50 centimètres de sa voûte» (d'Achon ibid.). Une photographie, prise peu après le début des fouilles de l'époque, montre encore une partie des terres qui masquaient l'entrée de la grotte, considérablement réduite par rapport à la masse d'origine.

\subsection{2 - Les profils du versant}

Nous avons relevé au tachéomètre trois profils (fig. 20) à l'emplacement des quatre locus archéologiques du site : le premier (orange) relevé à la hauteur de la grotte La Roche- 


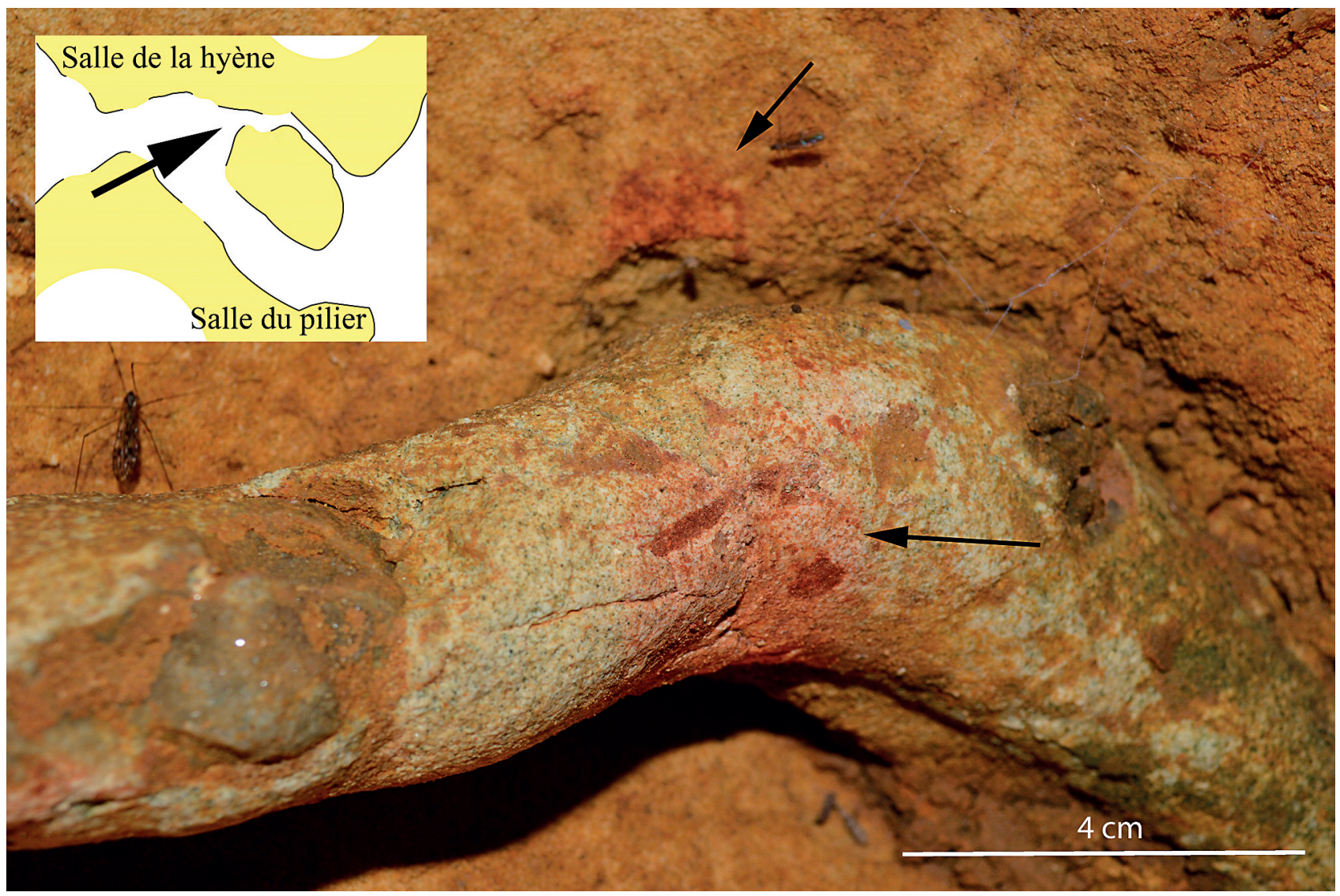

Figure 18 - La Roche-Cotard I. Deux taches ocre rouge dans le boyau qui conduit à la salle de la hyène. En haut, à gauche, la localisation de la niche qui contient trois ponctuations.

Figure 18 - La Roche-Cotard I. Two red ochre stains in a niche in the tunnel which is situated between "la salle du pilier " and "la salle de la hyène ". Above and on left, localisation of the niche with three punctuations.

Cotard I et II, le deuxième (bleu) relevé au niveau de LRC III, le troisième au niveau de LRC IV (vert). Le profil du versant en dehors de la zone d'emprunt de terre (rouge) se distingue nettement des trois autres par une inflexion convexe (en B) due à la présence de la paroi de craie tuffeau qui apparaît aujourd'hui à l'affleurement au niveau de l'entrée de LRC I mais qui reste profondément enfouie au niveau de LRC IV. Les parties moyenne (BC) et basse (CD) de la zone hors emprunt donnent une forme que l'on peut qualifier de profil d'équilibre, se raccordant, aux alluvions modernes de la Loire, à une altitude proche de 42 mètres NGF, au niveau de la limite du parc, le long de la route de Cinq-Mars-la-Pile.

Au niveau du tiers supérieur $(A B)$ du versant, les trois profils de la zone d'emprunt sont pratiquement confondus. À la hauteur de LRC I, ils se raccordent avec le sommet du surplomb qui se trouve au-dessus de l'entrée de la grotte. Au niveau de LRC III et IV, deux mètres d'épaisseur de sédiment séparent encore le haut de la paroi calcaire de la surface actuelle du versant. Nous attribuons, en ces trois endroits, le manque de sédiment, non pas à l'emprunt direct de terre par les terrassiers modernes car nous sommes très haut sur le versant, mais plutôt à une régularisation du profil du versant, postérieure à l'emprunt, par le jeu du ruissellement et de la gravité, les ressauts rocheux, notamment celui de LRC I, empêchant un écoulement facile de la terre vers le bas. Ce sont d'ailleurs ces encorbellements rocheux qui ont considérablement freiné l'avancée des terrassiers alors que quelques vingt mètres plus à l'ouest, l'emprunt de terre a été beaucoup plus considérable et a donné un haut de versant en terre, encore aujourd'hui infranchissable à pied car trop abrupt.

Au niveau du tiers moyen $(B C)$ du versant, les profils de LRC III et IV (vert et bleu) rejoignent le profil d'équilibre (rouge). Pour LRC III, ceci est dû à la présence du massif de chert qui forme le toit de l'abri et vraisemblablement également la falaise (non observée) qui se trouve à l'aplomb du toit de l'abri. L'emprunt de terre a ici été poussé au maximum jusqu'à atteindre vraiment la verticale du front de l'abri, sa limite ayant été observée au moment de la fouille de ce locus. Pour LRC IV, il restait encore une certaine marge pour les terrassiers avant d'atteindre la paroi de tuffeau car il y a ici décrochement de la falaise vers le nord, le toit de LRC III formant une avancée importante vers le sud. On peut alors supposer qu'ayant été bloqués 


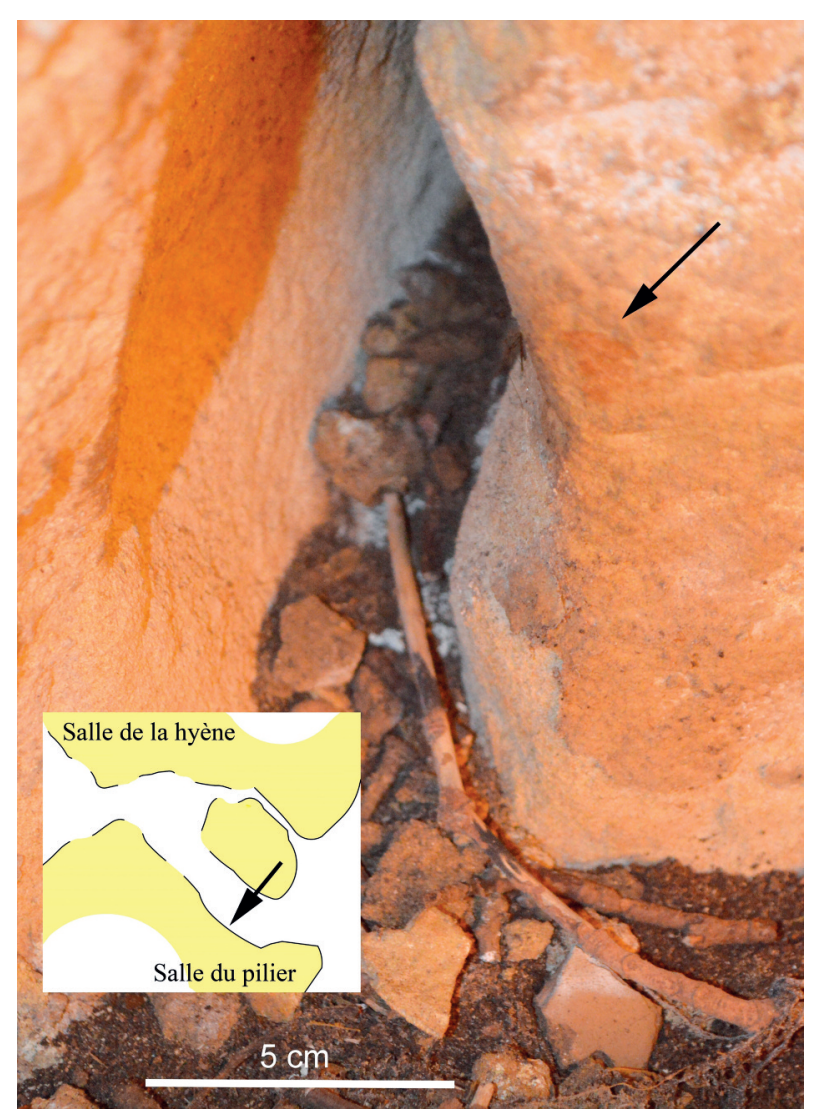

Figure 19 - La Roche-Cotard I. Tache ocre rouge et sa localisation dans le boyau qui conduit à la salle de la hyène.

Figure 19 - La Roche-Cotard I. Red ochre puncture and its localisation in the tunnel which conduct to "la salle de la hyène ».

dans leur avancée par la proéminence de l'abri, les terrassiers n'ont pas insisté, considérant qu'au niveau de LRC IV la paroi était proche.

Pour ce qui est du profil orange, à l'entrée de la grotte, l'emprunt de terre est très important puisqu'il met au jour, ou presque, non seulement l'espace qui se trouve devant l'entrée mais également tout l'espace qui longe la falaise de tuffeau vers l'ouest, jusqu'à l'endroit où cette falaise montre un décrochement vers le nord, pour arriver à une sorte de nouveau petit cirque rocheux semblable à celui qui se trouve devant l'entrée de LRC I. Lorsque d'Achon découvre la grotte, il ne reste plus qu'une ouverture de la taille d'un terrier et il semble que les terres atteignant alors le sommet de l'entrée sont soit des terres non affectées par l'emprunt, soit des terres accumulées, postérieurement, par gravité.

Au niveau du tiers inférieur (CD) du versant, les trois profils du site, très proches, s'écartent fortement du profil d'équilibre (rouge) du versant. La masse de matériau emporté est facilement repérable et pourrait même être quantifiée. Le fait que les trois profils de La Roche-Cotard I/II, III et IV coïncident globalement tient au fait que les terrassiers ont cessé de prendre des matériaux dès qu'ils ont rencontré un premier obstacle, en l'occurrence le toit et la base de LRC III. Devant LRC I le décaissement a été très important jusqu'à la rencontre de la falaise vers le nord. Vers l'ouest, l'extraction a été très poussée le long de la falaise et ensuite très loin.

À retenir de cette analyse des profils du versant, la totale occultation des entrées de la grotte (LRC I) et de la grotteabri (LRC IV) par des dépôts de versant en place jusqu'en 1856, ce qui a permis la découverte en 1912. Depuis, à l'exception de très rares visiteurs (une seule inscription moderne), l'accès de la grotte était uniquement protégé par sa situation dans une propriété clôturée de murs, celle-ci a été fréquentée par les ouvriers de F. d'Achon (traces de coups de pics à la base de certaines parois et au sol devant l'entrée du boyau) et par les équipes de fouilles des années 1970 et 2000. II n'est pas possible d'imaginer que ce sont les ouvriers de d'Achon qui ont laissé ces traces appliquées, rythmées, placées sur certaines parties choisies des parois. II en est a fortiori de même pour les quatre ponctuations rouges.

II convient enfin de s'interroger sur la date de la mise en place de ces dépôts de pente pour tenter d'avancer une hypothèse quant à l'âge de ces tracés. Pour traiter ce point chronologique, il nous faut revenir sur la stratigraphie et la chronologie des sédiments qui ont constitué le remplissage de la cavité.

\section{2 - Les remplissages}

La cavité LRC I est creusée dans la craie tuffeau sous la base du Sénonien, entre les altitudes de 48 et 52 mètres NGF. La figure 21 donne le profil en long de la partie proximale de la cavité selon le tracé de $\mathrm{E}$ à $\mathrm{H}$. En approchant de l'entrée de la grotte, le profil s'affaisse légèrement et montre un léger surcreusement guidé par les importantes masses cherteuses présentes.

Grâce aux observations stratigraphiques qui ont été faites sur des lambeaux de couches et qui ont été présentées plus haut, on peut replacer, au niveau de l'entrée de la grotte, la stratigraphie de la manière suivante :

- à la base, devant l'imposante masse cherteuse de l'entrée, la couche inférieure (i) qui contenait le riche niveau moustérien. Elle recouvrait la cuvette foyer dessinée sur le plan de d'Achon, et est toujours visible aujourd'hui (son point le plus profond est à $48,03 \mathrm{~m}$ ). Elle atteignait la base du lambeau de couche moyenne $(\mathrm{m})$ encore présent sous la dalle de chert inclinée de la paroi extérieure proche de l'entrée à 48,72 m. Sa puissance est de l'ordre de $50 \mathrm{~cm}$ puisque la profondeur du foyer est proche de $20 \mathrm{~cm}$;

- au-dessus, se trouve la couche moyenne (m), épaisse et hétérométrique d'un probable limon de débordement de la Loire (Macaire in Marquet 1997 - p.34). Sa base n'a été véritablement observée que sous la dalle inclinée citée précédemment où nous avons mis en évidence un éclat Levallois et un artefact sphérique, marquant le sommet de la couche inférieure (i). Le sommet de ce limon de débordement, si on considère qu'il n'a pas subi d'érosion 


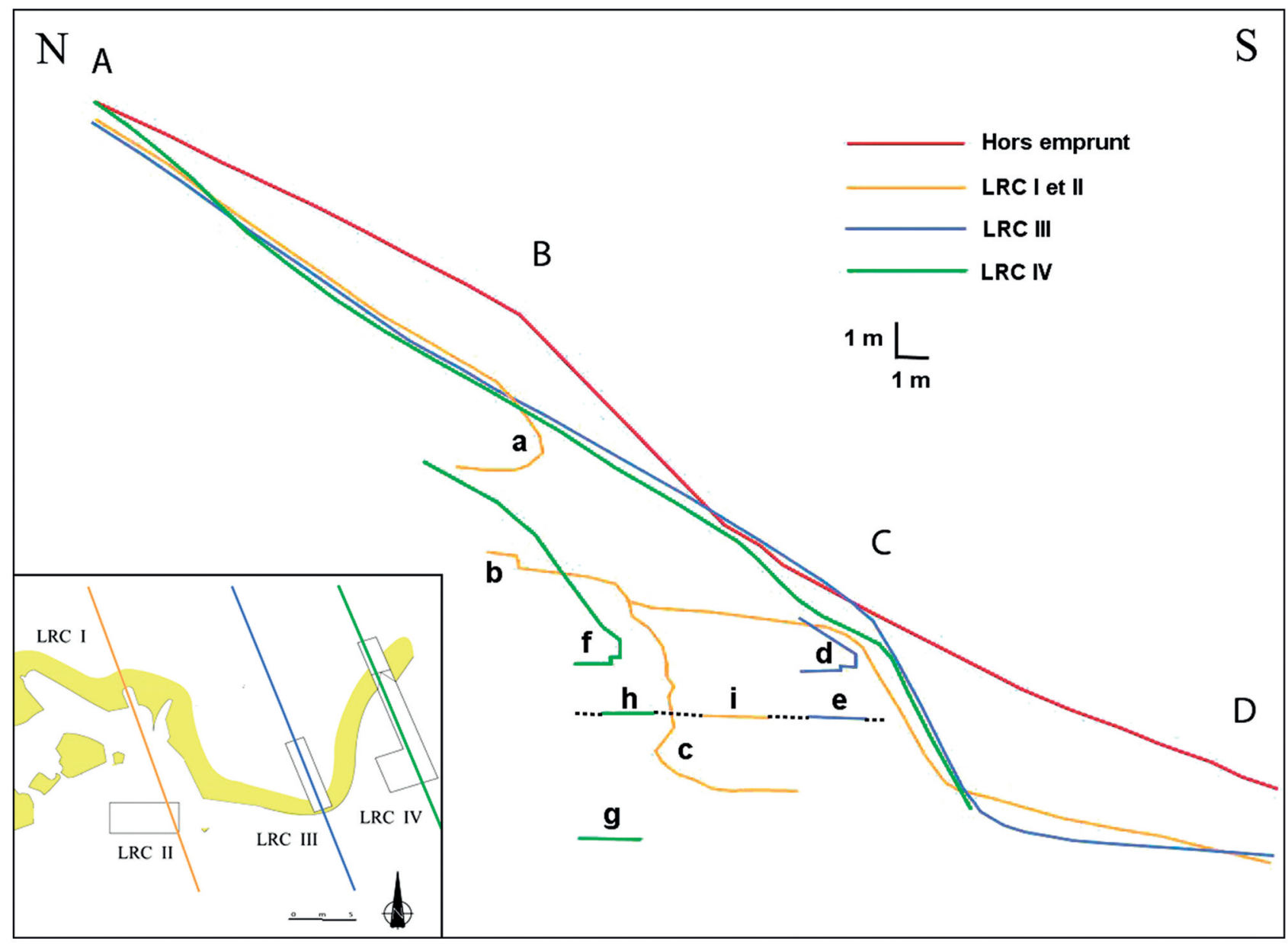

Figure 20 - Profils du versant du site de La Roche-Cotard. Rouge, le profil du versant en dehors de la zone de l'emprunt de terre de 1846. Orange : profil actuel au niveau de LRC I et LRC II. Bleu : profil actuel au niveau de LRC III. Vert :! Profil actuel au niveau de LRC IV. a: front de l'entrée de $L R C$ I; b. base de l'entrée de $L R C$ I; $c$ : base de la paroi sur laquelle s'appuie $L R C$ II; d: front de $L R C$ III; e: plancher de LMRC III constitué par le banc de calcaire dur; f: front de LRC IV; g: plancher de LRC IV; h: banc de calcaire dur dans LRC IC; i: banc de calcaire dur dans $L R C$ II. Les lettres $A, B, C$ et $D$ délimitent les trois parties, supérieure (AB), moyenne (BC) et inférieure $(C D) d u$ versant. En bas, à gauche, localisation des trois profils sur le site proprement dit. Le profil de la zone hors emprunt a été relevé à 100 mètres à l'ouest de la grotte.

Figure 20 - Profiles of the slope of the site of La Roche-Cotard. Red : the profile outside the place of extraction of materials in 1846. Orange : Actual profile at the LRC I and LRC II level, Blue : actual profile at the LRC III level, Green : actual profile at the LRC IV level. a: front of the entrance of $L R C$ I; b. base of the entrance of $L R C$ I; $c$ : base of the wall on which leans $L R C$ II; d: front of $L R C$ III; e: floor of $L R C$ III formed by the bed of hard limestone; f: front of LRC IV; g: floor of LRC IV; $h$ : bed of hard limestone in LRC IC; $i$ : bed of hard limestone in $L R C$ II. The letters $A, B, C$ et $D$ indicate the limits of the three parts, upper $(A B)$, medium $(B C)$ and lower $(C D)$ of the slope. Down, on left, localisation of the three profiles of the site.

avant le dépôt de la couche supérieure qui le recouvre, a été observé dans le passage P2 (fig. 21) qui fait communiquer la salle des lemmings avec l'extérieur, son altitude est de $50 \mathrm{~m}$ NGF. Son épaisseur totale est donc de l'ordre de 1,30 m ;

- au-dessus de ce limon de débordement, tranchant très nettement avec lui, la couche supérieure (s) a été retrouvée dans le même passage P2. Elle est encore présente, en place, sur le site. Elle ne contient aucun artefact mais est riche en restes fauniques et surtout en microfaune, essentiellement des rongeurs qui confirment le caractère très froid de l'épisode climatique (lemming à collier, campagnol des hauteurs). À propos de la nature du sédiment, J.-J. Macaire (In Marquet 1997 - p. 35) note qu'il s'agit d'un : "sédiment argilo-sableux...contenant de nombreux fragments de chert, souvent anguleux et aplatis. L'aspect des grains de quartz...et la granulométrie moyenne du sable concordent pour une origine éolienne bien que le classement soit mauvais". Le même auteur conclut :"...une part du sédiment (est) issu de la grotte ellemême, en particulier la cristobalite et les nombreux grains et plaquettes de chert qui ont été générées par 


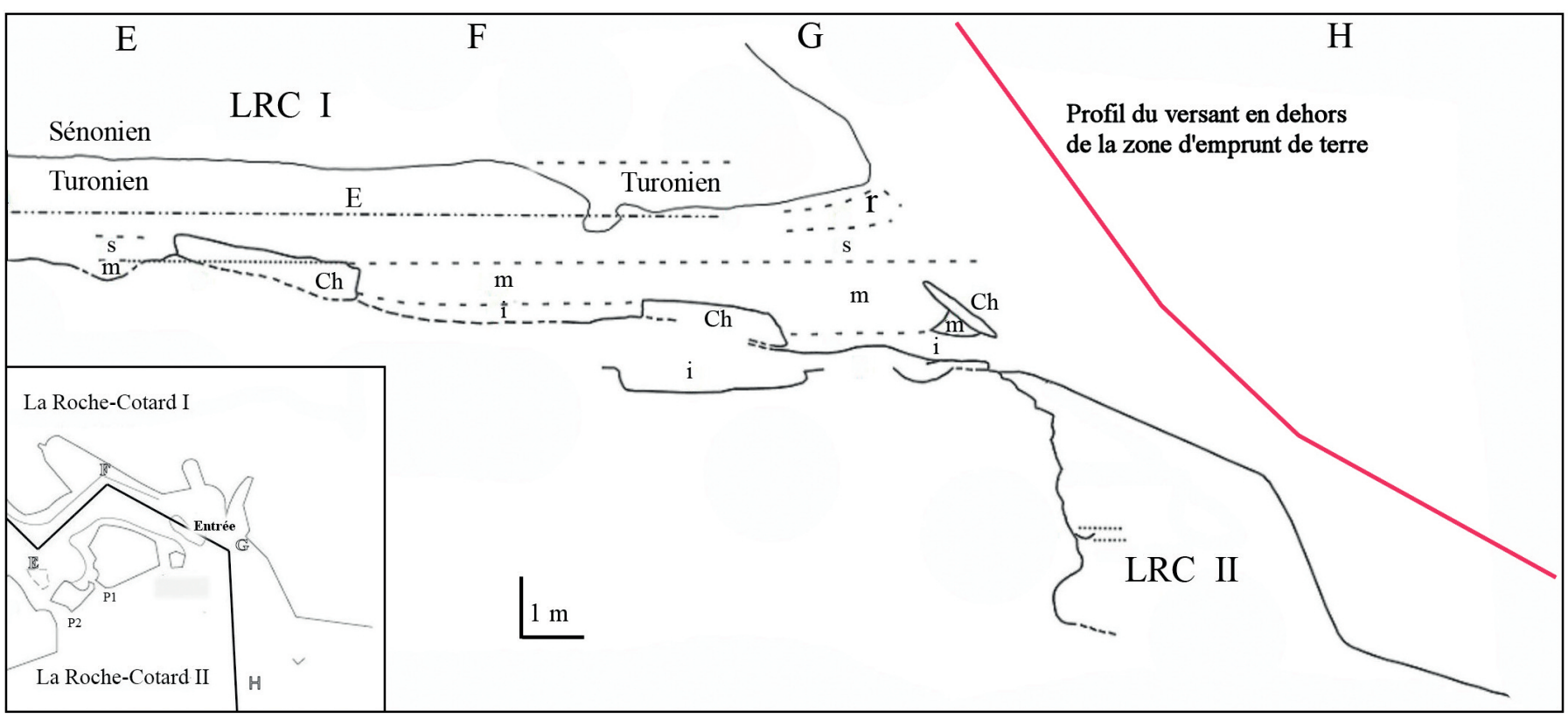

Figure 21 - La Roche-Cotard I. Profil longitudinal de la cavité présenté selon le tracé du plan en bas à gauche. Remplissage sédimentaire : i: couche inférieure; m: couche moyenne; s: couche supérieure; r: remanié. Ch : Chert turonien. $E$ : entaille en marche d'escalier renversée due à la dissolution de la craie par l'eau qui a stagné à l'intérieur de la cavité sur une très longue période et avec un système de trop-plein.

Figure 21 - La Roche-Cotard I. Adapted longitudinal profile of the cavity according to the plan on left. Sedimentary filling : i: inferior layer; m: middle layer; s: superior layer; $r$ : reworked; Ch. Turonian Chert; $E$ : cleft in the form of inverted stairs due to the water that has stagnated on the inside of the cavity for a very long period and with an overflow system.

gélifraction...le reste a été soufflé depuis les alluvions de la Loire situées à l'extérieur de la grotte"... La présence de très nombreux restes de rongeurs confirme cette provenance externe, les rapaces qui ont abandonné ces restes devant habiter la paroi au-dessus de ce conduit. Le sommet de cette couche, à l'intérieur de la salle des lemmings est de 50,58 m. Cette couche a été datée sur un os par le ${ }^{14} \mathrm{C}$ de 34795 à 35942 av. J.-C. cal. (Lyon 6962) et, sur le sédiment, de $64100 \pm 5100$ par la méthode OSL (MFGI Budapest).

S'il est difficile de connaître l'extension, aussi bien vers l'intérieur que vers l'extérieur de la cavité, de la couche supérieure (à éléments cryoclastiques et à vestiges fauniques de climat froid) en revanche, il est certain que ce limon de débordement de la couche moyenne devait naturellement s'étendre très largement devant la cavité. Ce même limon de débordement est présent dans le remplissage de La Roche-Cotard III dont il constituait la plus grande part mais à une altitude bien inférieure à celle du dépôt dans LRC I. En revanche, LRC IV, sensiblement à la même altitude que dans LRC III, contient des dépôts de type fluviatile un peu différents du limon de débordement mais avec des figures de décantation (présence de lits particulièrement minces dont certains composés presque exclusivement de paillettes de mica issues des alluvions de la Loire) qui n'en sont peut-être pas tellement éloignées.
Cette couche de limon a par la suite été érodée soit par de nouveaux débordements de la Loire, soit tout simplement par l'érosion due aux eaux ruisselant sur le versant. Au cours d'une, ou plus vraisemblablement de plusieurs phases climatiques ultérieures, des matériaux probablement soliflués (sédiments issus du plateau en grande partie, très hétérométriques, composés d'une matrice riche en sables et en argiles et contenant, sans stratification ni autre organisation, des éléments de taille variée et pluricentimétrique) vont se mettre en place sur le versant. Ainsi, pendant toute la période d'occupation des quatre locus du site dont la durée est difficile à estimer, la Loire était proche du versant, déposant même à son pied des alluvions (LRC II et IV mais vues aussi, un peu plus en amont grâce à un forage effectué par le SRA - Marquet ibid). Par la suite, à la faveur d'épisodes climatiques froids, dont la couche supérieure de La Roche-Cotard I est un indice, les matériaux soliflués depuis le rebord du plateau vont complètement ennoyer le versant.

\section{3 - Les datations}

Les dépôts de pente de La Roche-Cotard IV masquent encore aujourd'hui l'entrée de la grotte-abri. Ces formations ont été datées par le ${ }^{14} \mathrm{C}$ grâce à de très rares os qu'elles ont livrés (fig. 22). L'un deux, provenant du sommet de la couche $7 \mathrm{a}$ (D1), trouvé au niveau du carré $\mathrm{Q9}$, à une altitude de $46 \mathrm{~m}$ a donné un âge de 31048 à 29486 ans av. J.-C. cal. (Lyon 9087). II ne date pas le début de la mise 


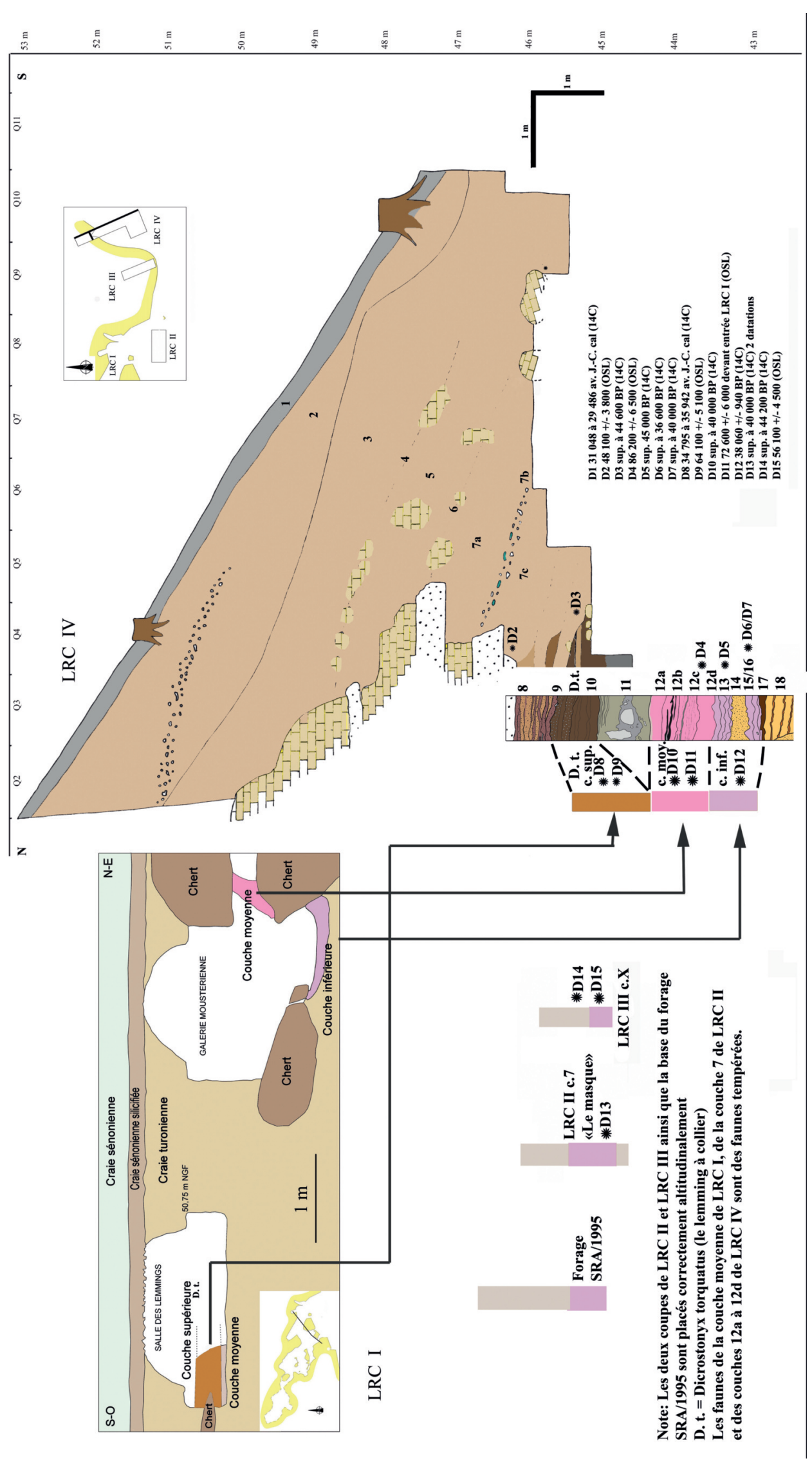

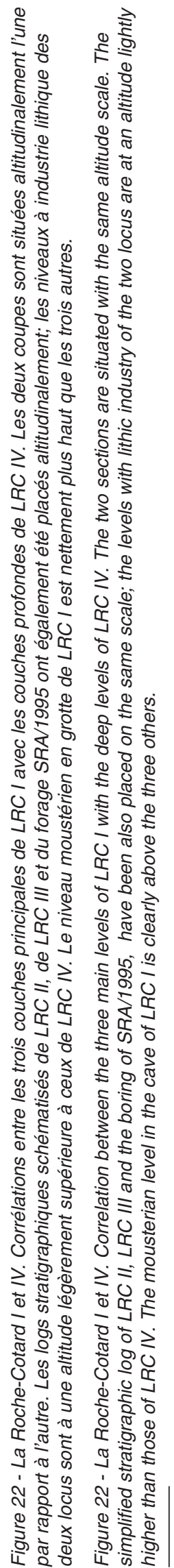


en place de ces solifluxions puisqu'il se situe géométriquement environ à «mi-parcours» de ce dépôt. Une datation par la méthode OSL de sédiment proche de la base de la couche 7c (D2), prélevé dans le carré Q5, a donné un âge de $48100 \pm 3800$ ans (MFGI Budapest). Une autre datation ${ }^{14} \mathrm{C}$ sur un os provenant du sommet du remplissage, à l'entrée de la grotte-abri (D3), dans la couche 9 (au niveau du carré R4), confirme une date supérieure à 44600 ans (Lyon 10 160).

Ces datations permettent d'affirmer qu'au niveau de La Roche-Cotard IV :

- le remplissage de la grotte-abri a atteint le plafond vers 44000 ans ou même avant ;

- une phase d'érosion va enlever toute la partie des couches 2 à 9 qui se trouvent en avant du front de la grotteabri ;

- les dépôts sur le versant vont se mettre en place à partir d'au moins 40000 ans.

Vers 30000 ans, l'entrée de la grotte-abri était déjà occultée depuis longtemps, les colluvions continuent de se mettre en place pour masquer complètement la falaise turonienne.

Devant l'entrée de la grotte d'Achon (LRC I), nous avons pu dater la couche moyenne (limon d'inondation) grâce à un lambeau protégé au fond d'une petite niche (D11). La datation par la méthode OSL donne un âge de $72600 \pm$ 6000 ans (MFGI Budapest) ; la mesure radiocarbone ne nous est pas encore parvenue. En revanche les datations ${ }^{14} \mathrm{C}$ de la même couche, à l'intérieur de la grotte, ont donné un âge supérieur à 40000 ans (Lyon 6961) pour un os provenant du passage P2 de la salle des lemmings vers l'extérieur de la cavité (D10) et $38060 \pm 940$ BP (Lyon 7864) pour un os provenant d'une niche de la galerie moustérienne. Sur la même couche, une précédente datation nous avait donné un âge supérieur ou égal à 38400 ans (Gif 4447).

Toujours pour La Roche-Cotard I, le lambeau de couche supérieure, dans le même passage P2 de la salle des lemmings vers l'extérieur, a été daté (D8) par le ${ }^{14} \mathrm{C}$ de 34 795 à 35942 av. J.-C. cal (Lyon 6962) alors que la méthode OSL (D9) a donné un âge de $64100 \pm 5100$ ans (MFGI Budapest).

Les datations des sédiments prélevés devant l'entrée de la grotte, dans son entrée et dans un conduit qui fait communiquer la grotte directement avec l'extérieur. Ces résultats permettent d'affirmer, pour La Roche-Cotard I que :

- la couche de limon d'inondation (couche moyenne) qui ferme en très grande partie l'entrée de la grotte, se met en place au plus tard entre 35 et 40000 ans ;

- la couche supérieure correspondant à un épisode climatique particulièrement froid, recouvre la couche sousjacente au plus tard vers 35000 ans, probablement avant une phase érosive importante (écoulement du fleuve au pied du versant) qui va emporter, comme devant l'abri LRC III et la grotte-abri LRC IV, la plus grande partie des matériaux déposés, hormis les sédiments piégés.

\section{7 - Les tracés digitaux de La Roche- Cotard : hypothèses et conclusion}

II y a 35 à 40000 ans, la grotte est largement colmatée de sédiments apportés par la Loire et déposés ensuite par vannage depuis les alluvions du fleuve qui coule juste en bas du versant et par l'apport d'éléments cryoclastiques issus des roches qui constituent la falaise et les parois de la grotte près de l'entrée. Lorsque d'Achon pénètre dans la cavité par un terrier, il ne subsiste, à l'entrée, qu'un passage de 20 à $30 \mathrm{~cm}$ de hauteur. La géométrie des dépôts de pente résiduels qui masquent la station de pied de paroi LRC II, l'abri LRC III et la grotte-abri LRC IV et celle du dépôt du versant actuel en dehors de la zone d'emprunt, nous conduisent à considérer qu'avant la période de décaissement, les dépôts résiduels et a fortiori l'entrée de la grotte d'Achon se trouvaient cachés par les matériaux prélevés. Les formations de versant qui scellent aujourd'hui la grotte-abri LRC IV (fig. 22) ont commencé à se mettre en place depuis au moins 35000 ans, comme nous l'indiquent les datations obtenues. Étant donnée la configuration du terrain, il est impossible que ces dépôts n'aient pas également affecté l'entrée de la grotte d'Achon qui était déjà emplie des sédiments préservés de l'érosion presque jusqu'à son plafond, dans toute sa partie proximale, de la même manière qu'ils ont ennoyé tout le versant comme on le constate aujourd'hui en dehors de la zone d'emprunt. En conclusion, la grotte a été fermée, il y a au moins 35000 ans, par les dépôts qui se sont accumulés presque jusqu'à son plafond, puis par les colluvions de versant qui l'ont complètement occultée.

Ce site complexe comprend au moins quatre locus habités par l'homme qui y a laissé des traces de son passage sous forme d'artefacts, de reliefs de repas, et d'installations à caractère utilitaire (cuvettes-foyers, aménagements de blocs). Aucun indice d'occupation par l'homme moderne n'a été retrouvé. En revanche l'homme de Néandertal signe sa présence par un outillage caractéristique (racloirs, bifaces triangulaires, débitage levallois, retouchoir sur os). Il est évident que les chasseurs moustériens ont fréquenté ce site à plusieurs reprises malgré l'absence sur place de matière première de qualité pour la production de leurs outils.

Nous avons présenté les principaux panneaux de tracés digitaux (ou réalisés avec un outil), les plus lisibles. Ces tracés, peut-être peut-on dire ces « décors ", n'ont pas été faits à n'importe quel endroit sur la paroi : même s'il est probable que certains panneaux ont été complètement effacés, les emplacements paraissent avoir été soigneusement choisis. C'est le cas par exemple du panneau triangulaire qui couvre une surface offerte par la morphologie de la paroi. Les tracés parallèles sont soignés, appliqués, rythmés, peut-être même réfléchis comme on le voit près du grand triangle de limon préservé où le doigt, à la différence des longues traces verticales faites d'un seul trait, semble avoir été passé lentement, par une série d'appuis un peu plus forts comme pour souligner le relief que l'on voulait préserver. Le soin avec lequel cette surface a été couverte de traces organisées, la préservation de 
certaines parties laissées en relief, la forme de cette surface, la présence du cylindre de chert à la pointe, en bas de ce triangle ne laissent pas de nous étonner et de nous interroger.

II faut rappeler l'absence totale de tout indice d'industrie postérieure au Paléolithique moyen dans les couches en place du site, absence logique étant donnée l'installation ancienne des dépôts de versant. Quant aux tracés et taches ocrées se trouvant dans la grotte, il est impossible d'envisager leur réalisation lors des travaux de 1912 : pour les traces d'ocre, en raison de leur emplacement difficile à atteindre ; pour les tracés, l'application avec laquelle ils ont été réalisés, l'altération qu'ils ont subie, tellement douce et progressive d'une extrémité du panneau à l'autre excluent toute réalisation récente.

En conséquence, nous ne pouvons que proposer l'hypothèse selon laquelle les tracés, de même que les quatre taches d'ocre rouge, ont été réalisés par l'homme de Néandertal, seul homme à occuper cette cavité avant sa réouverture en 1912. Si nous considérons bien le masque de La Roche-Cotard II comme une protofigurine, produite également par l'homme de Néandertal, aucun indice en revanche, ne nous permet d'avancer une quelconque contemporanéité de ces différentes productions à caractère symbolique.

\section{8 - Conclusion}

La recherche sur les débuts de l'art pariétal et rupestre ne cesse de progresser grâce à de nouvelles découvertes et à des rectifications d'erreurs et présupposés antérieurs. C'est ainsi par exemple que les parois couvertes de cupules de la grotte de Daraki Chattan (Madhya Pradesh) attribuées à l'Acheuléen par G. Kumar et R. Bednarik se sont avérées indatables lors d'un récent examen du site, la grotte ayant été envahie par des éboulis provenant de la surface, les quelques rares vestiges archéologiques que contenaient ces dépôts exogènes étaient sans rapport certain avec les cupules pariétales (Lorblanchet 2013).

La tradition des cupules a cependant une origine largement antérieure au Paléolithique supérieur. La sépulture néandertalienne $n^{\circ} 6$ de La Ferrassie, aujourd'hui datée des environs de 50000 ans était recouverte d'une pierre à cupules. Sur le même site, cette tradition se perpétue régulièrement dans les niveaux aurignaciens sus-jacents où elle donne naissance à un art figuratif sur blocs puis à des gravures par cupules sur paroi de grotte, comme par exemple à l'Abri de Belcayre en Dordogne et à la grotte des Fieux dans le Lot (Lorblanchet 1999, 2006). Par ailleurs, les datations $U$-Th de ponctuations et mains négatives de onze grottes espagnoles (dont Castillo, Altamira, Tito Bustillo) obtenues en 2012 par l'équipe de AWG. Pike et D. Hoffmann (Pike et al. 2012 ; Pike et Petitt 2012) confirment l'allongement de la chronologie de l'art paléolithique qu'indiquaient déjà les datations AMS. Nous sommes revenus aujourd'hui (sans surprise pour certains) aux «400 siècles d'art pariétal» du titre du fameux livre de H.Breuil publié en 1952.
Ces dates U-Th mettent l'accent sur la longue durée de l'art pariétal, c'est-à-dire sur une probable hétérogénéité des formes et des contenus, conduisant à l'acceptation de ruptures au sein d'une évolution stylistique que l'on avait cru univoque avec montée progressive vers l'apogée du naturalisme. Déjà Chauvet, Cussac, Lascaux, Altamira, l'art de plein air du Côa, nous apparaissaient comme des chefs d'œuvres absolus, dans un splendide isolement, s'intégrant difficilement dans une évolution stylistique globale... la question que posent à nouveau les dates U-Th est celle de l'existence d'une évolution unique ou d'un kaléidoscope stylistique pendant l'immense durée de l'art pariétal.

Les datations de certains décors pariétaux au-delà de 35000 ans et autour de 40000 ans - confirmée encore récemment en Indonésie (Aubert et al. 2014) correspondent, en Europe, à la période de contemporanéité de l'homme de Néandertal et de l'homme moderne ; la question de la paternité de l'art pariétal européen est ainsi clairement posée : l'art pariétal le plus ancien est-il exclusivement l'œuvre de l'homme moderne ? N'est-il pas parfois l'œuvre de l'homme de Néandertal ? Certes, les méthodes de datation par Uranium-Thorium sont encore à un stade expérimental qui peut avoir des limites (White et al. 2012 ; Pons-Branchu et al. 2014), mais elles connaissent maintenant d'importants progrès et ouvrent un horizon immense à la datation de toutes les formes d'art rupestre et pariétal. Elles doivent donc être encouragées. La découverte récente d'un signe pariétal gravé de la grotte de Gorham à Gibraltar (Kambiz Kamrani 2014), semble indiquer par ailleurs, l'existence de gravures pariétales moustériennes. Les données archéologiques et géologiques de La Roche Cotard apportent donc des éléments nouveaux qui vont dans la même direction... que les hommes de Néandertal puissent être les auteurs de certains tracés digitaux non figuratifs, tout comme de ponctuations à l'ocre rouge ou de certaines mains négatives, ou de tracés gravés, ce sont des hypothèses qui s'imposent aujourd'hui : l'ancien paradigme d'une apparition de l'art pariétal en occident liée à l'avènement d'une nouvelle espèce humaine peut, désormais, être mis en doute.

Remerciements à Philippe Brulé et Dimitry Ferey, géomètres-topographes au Syndicat Cavités 37, aux relecteurs de la revue Paleo pour leurs remarques et conseils, à J.-J. Macaire et $\mathrm{H}$. Guillemot pour leurs relectures du texte ainsi qu'à $M$. et Mme Bernard Menassanch, propriétaires de la grotte et à Mme Marcelle Menassanch.

Les travaux sur le site sont financés par l'État (DRAC /SRA Centre), le Conseil régional du Centre (Association ARCHEA) et la société Cofiroute.

\section{Références bibliographiques}

ACHON (d') F. 1913 - Au pays des châteaux. La RocheCotard. Sa grotte préhistorique. La Touraine $1^{\text {ère }}$ année, $\mathrm{n}^{\circ} 4$. 
AJAS A. 2011 - Étude géoarchéologique du gisement paléolithique de La Roche-Cotard I et IV (Langeais, Indreet-Loire). Rapport inédit. PACEA, UMR 5199, Univ. Bordeaux 1, $28 \mathrm{p}$.

AUBERT M., BRUMM A. RAMLI M., SUTIKNA T.D., SAPTOMO E.W., HAKIM B., MORWOOD M.J., VANDENBERGH G.D., KINSLEY L., DOSSETO A. 2014 Pleistocene cave art from Sulawesi, Indonesia 9 oct. 2014 Nature vol 514 p. $222-240$.

AUJOULAT N. et CHEVILLOT C. 1989 - Une découverte exceptionnelle à Saint-Martin-de-Fressengeas (Dordogne) : la grotte des Fraux, Documents d'Archéologie et d'Histoire Périgourdines, 4, p. 39-44, 2 fig.

BALLARD C. 1992 - First Report of Digital Fluting from Melanesia. Rock Art Research 9, n² 2, 1992, p. 119-121.

BEDNARIK R. 1986 - Parietal finger Markings in Europe and Australia. Rock Art Research, volume 3, n 1, p. 30-61.

BEDNARIK R. 1994 - The Discrimination of Rock Markings. Rock Art Research, 1994, volume 11, n 1, p. 23-44.

BEDNARIK R. 2014 - The known cave art sites of Australia. Voir : http://www.ifrao.com/wp-content/uploads/

2014/06/Cave table.doc

BYRNE N., DELPECH F., PRAT F. 1997 - La RocheCotard. Répartition des restes osseux. In : Marquet J.-C. 1997, p. 37.

DUVALL D. 2010 - Finger fluting and other cave art in Cumayasa, Dominican Republic Rock Art Research, 2010, volume $27, n^{\circ} 2$, p. $1-10$

KANBIZ KAMRANI 2014 - 40000 years old Neanderthal Hashtag Engravings from Gorahm's cave in Gibraltar; Anthroplogy.net 2 sept. 2014

LORBLANCHET M. 1995 - Les grottes ornées de la préhistoire. Nouveaux regards. Paris : Éditions Errance, $288 \mathrm{p}$.

LORBLANCHET M. 1999 - La Naissance de l'Art, genèse de l'art préhistorique. Paris, Editions Errance, 304 p.

LORBLANCHET M. 2006 - Les Origines de l'Art, Paris, Éditions du Pommier, Cité des Sciences, $187 \mathrm{p}$.

LORBLANCHET M. 2008 - Visite à la grotte de La RocheCotard (Indre et Loire). Rapport inédit. 5 p. In Rapport d'opération de sondage. Marquet J.-C. 2008, Service régional de l'archéologie du Centre.

LORBLANCHET M. 2013 - Les débuts de l'art rupestre dans le monde, In : Colloque de l'École Pontificale des Sciences, Rome 2013, à paraître aux éditions du CNRS.

MAN-ESTIER E., PAILLET P. 2009 - Rapport d'étude. La Roche-Cotard. Inédit 2 p. In : Marquet J.-C. 2009, Rapport de fouille programmée. Service régional de l'archéologie du Centre.
MARQUET J.-C., BYRNE N., DELPECH F., MACAIRE J.-J., PRAT F. et RAGE J.-C., 1979 - Un grand site préhistorique en Touraine. La Roche-Cotard à Langeais. Grotte et station du paléolithique moyen. Laboratoire de Géologie. Faculté des sciences. Université François Rabelais, Tours, 1979, 175 p. 64 fig.

MARQUET J.-C., AUBRY T., BYRNE N., DELPECH F., DESSE G., MACAIREJ.-J., PRAT F., RAGE J.-C., URBAN B., VISSET L. 1997 - Le site préhistorique de La RocheCotard à Langeais (Indre-et-Loire). Ed. CLD Chambray-lesTours, 127 p., 82 fig, 24 tabl., 6 pl. hors texte.

MARQUET J.-C., LORBLANCHET M. 2000 - Le masque moustérien de La Roche-Cotard, Langeais (Indre-et-Loire), Paleo, $\mathrm{n}^{\circ}$ 12, p. 325-338.

MARQUET J.-C., LORBLANCHET M. 2003 - A Neanderta face ? The protofigurine from La Roche-Cotard, Langeais (Indre-et-Loire, France), Antiquity volume 77, $\mathrm{n}^{\circ} 298$, p. 661-670.

PETROGNANI S., BOURRILLON R., BURENS A.,CAROZZA L. 2014 - A la poursuite du temps court : les expressions pariétales de l'âge du Bronze de la grotte des Fraux (Dordogne, France) Paleo numéro spécial 2014 p. 163 à 169.

PETZINGER G. von, NOWELL A. 2011 - A question of style: reconsidering the stylistic approach to dating palaeolithic parietal art in France, In : Antiquity, volume $85, n^{\circ} 330$, p. $1165-1183$.

PIKE A.WG., PETITT P. 2012 - Redating Ice Age Art; were Neandertals the first artists in Europe? Current World Archaeology, Issue 55, p.22-27

PIKE A.WG., HOFFMANN D., GARCIA-DIEZ M., PETITT PGB., ALCOLEA J., BALBIN R.de, GONZALES-SAINZ G., LAS HERAS C. de, LAS HERAS J.A., MONTES R., ZILHAO J. 2012 - U-series Dating of paleolithic Art in 11 caves in Spain, SCIENCE, vol 336, 15, june 2012, p.1409-1413

PONS-BRANCHU E., BOURILLON R., CONKEY M., FONTUGNE M., GARATE D., QUILES A., RIVERO O., SAUVET G., TOSELLO G., VALLADAS H., WHITE R. 2014 - Uranium-series dating of cartonate formations overlying Paleolithic art, interest and limitations. Bulletin de la Société préhistorique Française,2014-2, avril-juin p.211-224.

RIALLAND Y., DETRAIN L., LENOBLE A. 1995 - A-85. Contournement nord de Langeais (Indre-et-Loire). La Roche-Cotard. Rapport d'étude d'impact. Ministère de la Culture. Préfecture de la Région Centre. DRAC. Service régional de l'Archéologie. 46 p. 19 fig. 6 pl. photo.

SHARPE K. 2004 - Line Markings : Human or Animal Origin? in Rock Art Research, p. 57-84. 
SHARPE K., VAN GELDER L. 2004 - Les enfants et «l'art» paléolithique : indices à la grotte de Rouffignac, France, Inora, 38, p. 9 - 16. Voir http://www.international.icomos.org/centre_documentation/ inora/inora38/inora-38-3.pdf, (vu le 21/07/2014).

SHARPE K., VAN GELDER L. 2005 - Techniques for Studying Finger Flutings. Society of Primitive Technology Bulletin, 30, p. 68-74.

THAMO-BOZSO E. 2013 - Results of OSL dating La Roche-Cotard LRC/OSL/1-6 samples et Results of OSL dating La Roche-Cotard LRC/OSL/7 sample. Inédits, Magyar Fölsdtani és Geofizikai Intézet, Budapest, 16 p. et $6 \mathrm{p}$.

TOURON S., BOURGES A. 2012 - Langeais (37, Indre-etLoire). Grotte de La Roche-Cotard. Identification d'argile et concrétion blanches. Rapport d'étude inédit. Laboratoire de recherches des monuments historiques. Champs-surMarne.
VAN HOEK M. 2011 - Aldea de Ramaditas, Chile: Architectural Art or Rock Art? Voir http://www.rupestreweb.info/aldearamaditas.html (vu le 21/07/2014).

WHITE R., MENSAN R., BOURRILLON R., CRETIN C., HIGHAM T., CLARK E., SISK M., TARTAR E., GARDĖRE P., GOLDBERG P., PELEGRIN J., VALLADAS H., TSINERAT-LABORDE N., SANOIT (de) J., CHAMBELLAN D., CHIOTTI L. 2012 - Context and dating of Aurignacian vulvar representations from Abri Castanet, France, In : PNAS, T. $109, n^{\circ} 22$, p. $8450-8455$

WINTLE A., MURRAY A.S. 2006 - A review of quartz optically stimulated luminescence characteristics and their relevance in single-aliquot regeneration dating protocols. Radiation Measurements 41, 369-391. 